\title{
Transversal Sets
}

\author{
Milan R. TASKOVIĆ* \\ This paper is dedicated to professor D. Adamovic on his $77^{\text {th }}$ birthday.
}

\begin{abstract}
This paper presents a new concept of sets which we call it transversal (upper, lower or medial) sets. We introduce this concept as a natural extension of ordinary, fuzzy and transversal fuzzy sets. Transversal sets are a new way in the nonlinear analysis. Applications in minimax theory, algebra, topology, analysis, games theory, algebraic equations theory, ideal theory of BCC-algebras, theory of measures and integration, and convex analysis are considered. First time in history of the theory sets, we give a technology of an arbitrary set, in the sense that every set has "three sides" which are invisible but they de facto existing.
\end{abstract}

\section{Introduction, DEFinitions, And FUndamental faCts}

The mathematical concept of a set can be used as the foundation for all known mathematics facts. A flock of pigeons, or a bunch of grapes are examples of sets of things. Sets, as they are usually conceived, have elements or members. An element of a set may be a pigeon, or a grape; i.e., this means an atomistic classical admission sets. It is important to know that a set itself may also be an element of some other set. Mathematics is full of examples of sets of sets.

In the meanthime, there has developed a concept of the set-theory damaged school child, so we must ensure that this paradise remains a blooming garden and does not turn into rocky ground and thorny scrub. In this sense, our admission, for this problem in this paper, give a new paradise for set-theory.

The classes of objects encountered in the real or a concoction physical word do not have precisely defined conditions of membership. In 1965 Zadeh introduced the notion of a fuzzy set which is an answer on this indistinction. A fuzzy set is a class of objects with a continuum of grades of membership.

1991 Mathematics Subject Classification. Primary: 06F35, 03G25. Secondary: 94D05, 54H25, $54 \mathrm{E} 35$.

Key words and phrases. Transversal set, transversal (upper, lower or medial) fuzzy set, grade of membership, fuzzy set, minimax equality, minimax principle, statement of separation, games theory, convex transversal (upper, lower or medial) set, transversal (upper, lower or medial) BCC-ideal, BCC-algebra, Borsuk-Ulam theorem, transversal integrals, transversal points, zeros of algebraic equations, transversal logic, transversal topological spaces.

${ }^{*}$ Research supported by Science Fund of Serbia under Grant 1457. 
In connection with the preceding, in this section we introduce the concept of a transversal set as a natural extension of ordinary, fuzzy and transversal (upper or lower) fuzzy sets. A transversal set is a class of objects with two characteristics: first, with a continuum of grades of membership and second, with new signification (an annex) foreign or inside influence and affair.

Let $X$ be a nonempty set, let $L:=(L, \preccurlyeq)$ be a lattice by the order relation $\preccurlyeq$ and let $g$ be a mapping from $L$ into $L$. A transversal upper set $A$ in $X$ is characterized by a transversal membership (characteristic) function

$$
\sup \left\{f_{A}(x), g\left(f_{A}(x)\right\}: X \rightarrow L,\right.
$$

where $f_{A}(x): X \rightarrow L$. In this case, the value of $f_{A}(x)$ at $x \in X$ can be representing the grade of membership of $x$ in $A$, till the value of $g\left(f_{A}(x)\right)$ can be representing an event (foreign or inside) which is in connection with the set $A$ in $X$.

In connection with this, let $P:=(P, \preccurlyeq)$ be a partially ordered set and for $a, b \in P$ and $a \prec b$, the set (interval) $[a, b]$ is defined by

$$
[a, b]:=\{t: t \in P \text { and } a \preccurlyeq t \preccurlyeq b\} .
$$

If the lattice $L:=[a, b]$ for $a \prec b$, then we have a very typical example of the transversal membership characteristic function (1). A special case, in this sense, if $[a, b] \subset \mathbb{R}(a<b ; a, b \in \mathbb{R})$ is essential for further applications. Also, if $[a, b]=[0,1]$, then (1) reduces to the membership characteristic function which representing the transversal (upper) fuzzy set $A$ in $X$, which is introduced in Tasković [9].

If $L:=(P, \preccurlyeq)$, where $P:=(P, \preccurlyeq)$ is a totally ordered set, then $(1)$ reduces to the transversal membership (characteristic) function of the transversal upper set $A$ in $X$ of the following form

$$
\max \left\{f_{A}(x), g\left(f_{A}(x)\right)\right\}: X \rightarrow P,
$$

where $f_{A}(x): X \rightarrow P$.

Second interpretation can be that the value of $\sup \left\{f_{A}(x), g\left(f_{A}(x)\right)\right\}$ representing, an example, the grade of membership of $x$ in $A$. If $g(x)=x: I \rightarrow I:=[0,1]$, then $(1 \mathrm{~m})$ reduces to the membership function $f_{A}(x)$ which representing the fuzzy set $A$ in $X$. We notice, the domain of definition of $f_{A}(x)$ may be restricted to a subset $M$ of $X$, where in this case $g: M \rightarrow M$.

Example 1. Let $X$ be the real line $\mathbb{R}$ and let $A$ be a transversal upper set of numbers which are much greater than 1 . Then for $g: I \rightarrow I$ defined by $g(x)=1-x$ and $f_{A}(0)=1$, $f_{A}(1)=0, f_{A}(4)=0.02, f_{A}(10)=0.03, f_{A}(99)=0.81$ and $f_{A}(400)=0.93$, we obtain a characterization of the set $A$ in $\mathbb{R}$.

In connection with the preceding, if $(L, \preccurlyeq)$ a lattice with zero $\boldsymbol{O}$, then a transversal upper set $A$ in $X$ is empty if and only if its transversal membership (characteristic) function is identically $\boldsymbol{O}$ on $X$.

In further, let $g, r: L \rightarrow L$. Two transversal upper sets $A$ and $B$ are equal, denoted by $A=B$, if and only if $\sup \left\{f_{A}(x), g\left(f_{A}(x)\right)\right\}=\sup \left\{f_{B}(x), r\left(f_{B}(x)\right)\right\}$ 
for all $x$ in $X$. Also, $A$ is a subset of $B$ (or $A$ is smaller than or equal to $B$ ) if and only if

$$
\sup \left\{f_{A}(x), g\left(f_{A}(x)\right)\right\} \preccurlyeq \sup \left\{f_{B}(x), r\left(f_{B}(x)\right)\right\}
$$

for all $x$ in $X$, in symbols $A \subset B$.

The union of two transversal upper sets $A$ and $B$ with respective transversal membership functions is a transversal upper set $C$, denoted by $C=A \cup B$, whose transversal membership (characteristic) function is related to those of $A$ and $B$ by

$$
x \mapsto \sup \left\{f_{A}(x), f_{B}(x), g\left(f_{A}(x)\right), r\left(f_{B}(x)\right)\right\}
$$

for $x \in X$. Evidently, the union has the associative property, i.e., the following equality holds $A \cup(B \cup G)=(A \cup B) \cup G$ for arbitrary transversal upper sets $A$, $B$ and $G$ in $X$.

We notice, the union of $A$ and $B$ is the smallest transversal upper set containing both $A$ and $B$. Precisely, if $D$ is any transversal upper set which contains both $A$ and $B$, then it also contains the union of $A$ and $B$. Indeed, the union $C=A \cup B$ defined by (2) contains both $A$ and $B$, since

$$
\sup \left\{f_{A}, g\left(f_{A}\right)\right\} \preccurlyeq \sup \left\{f_{A}, g\left(f_{A}\right), f_{B}, r\left(f_{B}\right)\right\}
$$

and

$$
\sup \left\{f_{B}, g\left(f_{B}\right)\right\} \preccurlyeq \sup \left\{f_{A}, g\left(f_{A}\right), f_{B}, r\left(f_{B}\right)\right\} .
$$

On the other hand, if $D$ is any transversal upper set containing both $A$ and $B$, then we have

$$
\sup \left\{f_{D}, g\left(f_{D}\right)\right\} \succcurlyeq \sup \left\{f_{A}, r\left(f_{A}\right)\right\}
$$

and

$$
\sup \left\{f_{D}, g\left(f_{D}\right)\right\} \succcurlyeq \sup \left\{f_{B}, t\left(f_{B}\right)\right\},
$$

and hence $\sup \left\{f_{D}, g\left(f_{D}\right)\right\} \succcurlyeq \sup \left\{f_{A}, f_{B}, r\left(f_{A}\right), t\left(f_{B}\right)\right\}$, which implies that we have the following fact $C:=A \cup B \subset D$.
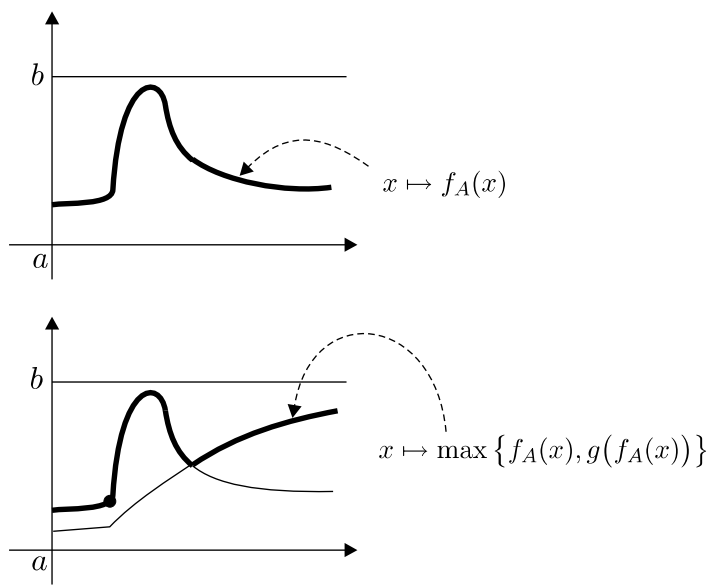

Figure 1

Analogous, the notion of an intersection of two transversal upper sets can be defined in the following sense. The intersection of two transversal upper sets $A$ and $B$ with respective transversal membership functions is a transversal upper 
set $C$, denoted by $C=A \cap B$, which transversal membership function is related to those of $A$ and $B$ by

$$
x \mapsto \inf \left\{f_{A}(x), f_{B}(x), g\left(f_{A}(x)\right), r\left(f_{B}(x)\right)\right\}
$$

for $x \in X$. As in the preceding case, it is easy to show that the intersection of $A$ and $B$ is the largest transversal upper set which is contained in both $A$ and $B$.

Also, $A$ and $B$ are disjoint if $A \cap B$ is empty. The intersection has the associative property, evidently. Otherwise, the complement of a transversal upper set $A$ in $X$ is denoted by $A^{c}$ can be defined as in the case of the ordinary sets. But, if the lattice $L:=[a, b] \subset \mathbb{R}$ for $a<b$, then the complement $A^{c}$ is defined with the following transversal membership function of the form

$$
x \mapsto b-\max \left\{f_{A}(x), g\left(f_{A}(x)\right)\right\}
$$

for $x \in X$. With the operations of union, intersection and complementation it is easy to extend De Morgan's and distributive laws which hold for ordinary and fuzzy sets.

We notice, that, if $X=\left\{x_{1}, \ldots, x_{n}\right\}$ for fixed $n \in \mathbb{N}$, is a finite set, then we have a very characteristic upper element (number) of $L$ as

$$
u\left(f_{A}, g\left(f_{A}\right)\right):=\min _{k=1, \ldots, n} \max \left\{f_{A}\left(x_{k}\right), g\left(f_{A}\left(x_{k}\right)\right)\right\},
$$

and, in case, if $X$ is an infinite set, then we have, also, a very characteristic upper element (number) of $L$, as

$$
u\left(f_{A}, g\left(f_{A}\right)\right):=\min _{x \in A \subset X} \max \left\{f_{A}(x), g\left(f_{A}(x)\right)\right\},
$$

or

$$
i u\left(f_{A}, g\left(f_{A}\right)\right):=\inf _{x \in A \subset X} \sup \left\{f_{A}(x), g\left(f_{A}(x)\right)\right\} .
$$

The upper transversal height of $A$, in notation $u[$ thgt $A)]$, is the following element of the form $u[\operatorname{thgt}(A)]=u\left(f_{A}, g\left(f_{A}\right)\right)$.

In connection with the preceding, let $X$ be a nonempty set, let $L:=(L, \preccurlyeq)$ be a lattice and let $g: L^{k} \rightarrow L, k$ is a fixed positive integer, i.e., $k \in \mathbb{N}$. An $k$-transversal upper set $A$ in $X$ is characterized by a transversal membership (characteristic) function

$$
\sup \left\{f_{A}\left(x_{1}\right), \ldots, f_{A}\left(x_{k}\right), g\left(f_{A}\left(x_{1}\right), \ldots, f_{A}\left(x_{k}\right)\right)\right\}: X^{k} \rightarrow L,
$$

where $f_{A}\left(x_{1}\right), \ldots, f_{A}\left(x_{k}\right): X \rightarrow L$. If $k=1$, then we have the case of a transversal upper set $A$ in $X$.

We notice, the operations of union, intersection and complementation analogous still hold when $g$ : $L^{k} \rightarrow L(k$ is a fixed positive integer), i.e., in the case of $k$-transversal upper sets. This operations are quite similar with the preceding facts.

If the lattice $L:=[a, b]$ for $a \prec b$, then we have a very typical example of the transversal membership characteristic function (3). A special case, in this sense, if $[a, b] \subset \mathbb{R}(a<b ; a, b \in \mathbb{R})$ is essential for further applications. Also, if $[a, b]=[0,1]$, then (3) reduces to the membership characteristic function which representing the $k$-transversal (upper) fuzzy set $A$ in $X$, which is introduced in Tasković [9]. 
If $L:=(P, \preccurlyeq)$, where $P:=(P, \preccurlyeq)$ is a totally ordered set, then $(3)$ reduces to the following transversal membership (characteristic) function of the $k$-transversal upper set $A$ in $X$ of the following form

$$
\max \left\{f_{A}\left(x_{1}\right), \ldots, f_{A}\left(x_{k}\right), g\left(f_{A}\left(x_{1}\right), \ldots, f_{A}\left(x_{k}\right)\right)\right\}: X^{k} \rightarrow P,
$$

where $f_{A}\left(x_{1}\right), \ldots, f_{A}\left(x_{k}\right): X \rightarrow P$. If $k=1$, then we have the case of a transversal upper set $A$ in $X$.

In analogous with the preceding facts, let $X$ be a nonempty set, let $L:=(L, \preccurlyeq)$ be a lattice and let $d$ be a mapping from $L$ into $L$. A transversal lower set $A$ in $X$ is characterized by a transversal membership (characteristic) function

$$
\inf \left\{f_{A}(x), d\left(f_{A}(x)\right)\right\}: X \rightarrow L,
$$

where $f_{A}(x): X \rightarrow L$. A transversal set $A$ in $X$ is a transversal upper and lower set, simultaneous. As an important example of transversal sets we have a Zadeh's fuzzy set in the case $g(x)=x: I \rightarrow I$.

If the lattice $L:=[a, b]$ for $a \prec b$, then we have a very typical example of the transversal membership characteristic function (4). A special case, in this sense, if $[a, b] \subset \mathbb{R}(a<b ; a, b \in \mathbb{R})$ is essential for further applications. Also, if $[a, b]=[0,1]$, then (4) reduces to the membership characteristic function which representing the transversal (upper) fuzzy set $A$ in $X$, which is introduced in Tasković $[9]$.

If $L:=(P, \preccurlyeq)$, where $P:=(P, \preccurlyeq)$ is a totally ordered set, then (4) reduces to the transversal membership (characteristic) function of the transversal lower set $A$ in $X$ of the following form

$$
\min \left\{f_{A}(x), d\left(f_{A}(x)\right)\right\}: X \rightarrow P,
$$

where $f_{A}(x): X \rightarrow P$.

An interpretation of transversal membership functions for transversal upper and lower sets we give in Figure 2 as parallel and series combinations of $f_{A}(x)$, $g\left(f_{A}(x)\right)$ and $d\left(f_{A}(x)\right)$, respectively. ${ }^{1}$

Some remarks. In the case new situations, a transversal (upper or lower) set is obviously an extension of an ordinary and a fuzzy set, and the transversal membership (characteristic) functions is a generalization of the membership functions of the ordinary and of the fuzzy set!

But our new concept of transversal sets (1) and (4) give manyfold and have an ambiguous in looking of the sets and in looking of the new calculation with them!

We notice, the transversal characteristic membership functions given in formulas (1) and (4) are not as the "standard" union and intersection, respectively, of the membership functions for the ordinary and the fuzzy sets!

The transversal (characteristic) membership functions (1) and (4), respectively, are essential different of the union and intersection fot the ordinary and the fuzzy sets!

\footnotetext{
${ }^{1}$ In this sense, we give a technology of an arbitrary set such that, de facto, every set has three sides which are invisible but they existing. Also, in a text of R ich ard Dedekind (1831-1916) spell: "I think of a set as a closed sack which contains certain specified objects which one doesn't see".
} 


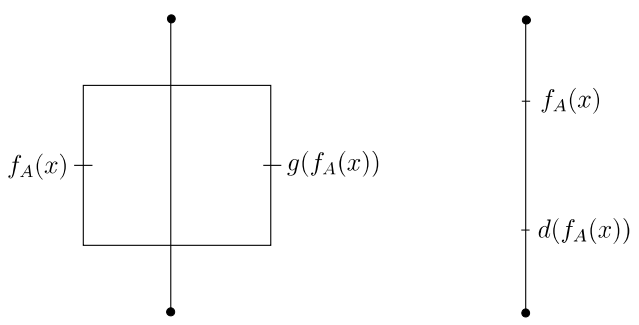

Figure 2

Let $X$ be a nonempty set, let $L:=(L, \preccurlyeq)$ be a lattice and let $d: L^{k} \rightarrow L$, where $k \in \mathbb{N}$ is fixed. An $k$-transversal lower set $A$ in $X$ is characterized by a transversal membership (characteristic) function

$$
\inf \left\{f_{A}\left(x_{1}\right), \ldots, f_{A}\left(x_{k}\right), d\left(f_{A}\left(x_{1}\right), \ldots, f_{A}\left(x_{k}\right)\right)\right\}: X^{k} \rightarrow L
$$

where $f_{A}\left(x_{1}\right), \ldots, f_{A}\left(x_{k}\right): X \rightarrow L$. If, in this case, $k=1$, then we obtain the case of a transversal lower set $A$ in $X$.

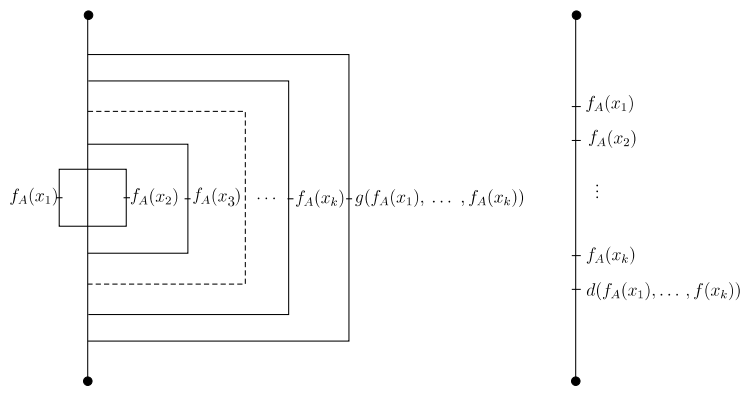

Figure 3

If the lattice $L:=[a, b]$ for $a \prec b$, then we have a very typical example of the transversal membership characteristic function (5). A special case, in this sense, if $[a, b] \subset \mathbb{R}(a<b ; a, b \in \mathbb{R})$ is essential for further applications. Also, if $[a, b]=[0,1]$, then (5) reduces to the membership characteristic function which representing the $k$-transversal (lower) fuzzy set $A$ in $X$, which is introduced in Tasković [9].

If $L:=(P, \preccurlyeq)$, where $P:=(P, \preccurlyeq)$ is a totally ordered set, then $(5)$ reduces to the transversal membership (characteristic) function of the $k$-transversal lower set $A$ in $X$ of the following form

$$
\min \left\{f_{A}\left(x_{1}\right), \ldots, f_{A}\left(x_{k}\right), d\left(f_{A}\left(x_{1}\right), \ldots, f_{A}\left(x_{k}\right)\right)\right\}: X^{k} \rightarrow P
$$

where $f_{A}\left(x_{1}\right), \ldots, f_{A}\left(x_{k}\right): X \rightarrow P$. If $k=1$, then we have the case of a transversal lower set $A$ in $X$. 

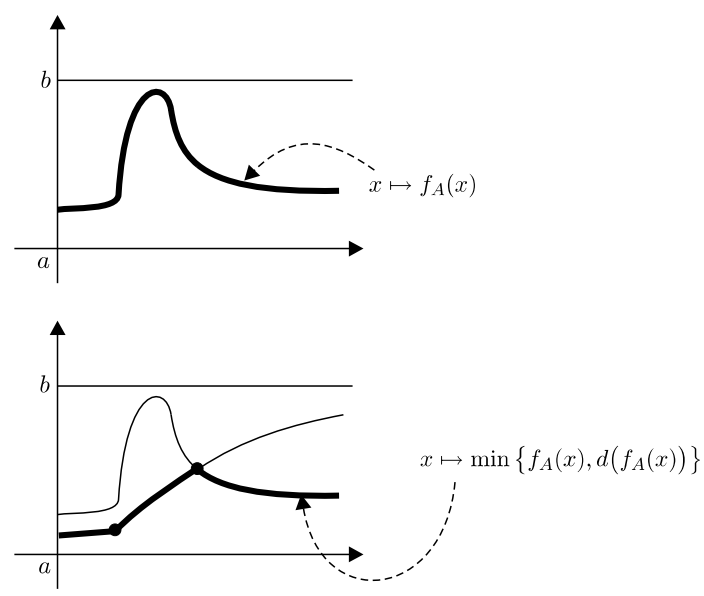

Figure 4

We notice, that the operations of union, intersection and complementation of transversal lower sets are totally analogous to defined, in comparable, with the analogous operations for transversal upper sets. Also, the domain of definition of $f_{A}(x)$ may be restricted to a subset $M$ of $X$, where in this case $d: M \rightarrow M$.

In this sense, if the lattice $L:=[a, b] \subset \mathbb{R}$ for $a<b$, then the complement of a transversal lower set $A$ in $X$ is denoted by $A^{\prime}$ and is defined with the following transversal membership function of the form

$$
x \mapsto b-\min \left\{f_{A}(x), d\left(f_{A}(x)\right)\right\}
$$

for $x \in X$. With the operations of union, intersection and complementation it is easy to extend De Morgan's and distributive laws which hold for ordinary, fuzzy and transversal (upper) fuzzy sets.

An interpretation of transversal membership functions for $k$-transversal upper and lower sets we give in Figure 3, respectively. Also, an interpretation of transversal (upper and lower) sets and fuzzy sets is an Figure 3.

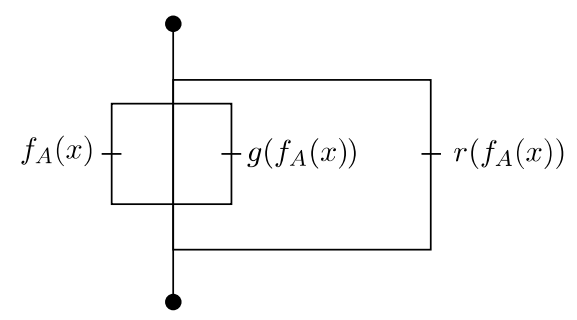

Figure 5

In analogy with the preceding facts, if $X=\left\{x_{1}, \ldots, x_{n}\right\}$ for fixed $n \in \mathbb{N}$, is a finite set, then we have a very characteristic lower element (number) of $L$ as

$$
l\left(f_{A}, d\left(f_{A}\right)\right):=\max _{k=1, \ldots, n} \min \left\{f_{A}\left(x_{k}\right), d\left(f_{A}\left(x_{k}\right)\right)\right\},
$$


and, in case, if $X$ is infinite set, then we have the following very characteristic lower element (number) of $L$ as

$$
l\left(f_{A}, d\left(f_{A}\right)\right):=\max _{x \in A \subset X} \min \left\{f_{A}(x), d\left(f_{A}(x)\right)\right\},
$$

or

$$
s l\left(f_{A}, d\left(f_{A}\right)\right):=\sup _{x \in A \subset X} \inf \left\{f_{A}(x), d\left(f_{A}(x)\right)\right\} .
$$

The lower transversal height of $A$, in notation $l[\operatorname{thgt}(A)]$, is the following element of the form

$$
l[\operatorname{thgt}(A)]=l\left(f_{A}, d\left(f_{A}\right)\right) .
$$

In connection with the preceding, a transversal upper or lower relation in $X$ is a transversal upper or lower set $A$ in the product set $X \times X$.

Analogoys, an $n$-ary transversal upper or lower relation in $X$ is an $n$-ary transversal upper or lower set $A$ in the product set $\underbrace{X \times X \times \cdots \times X}_{n-\text { ary }}$.

Example 2. Let $X$ be the real line $\mathbb{R}$ and let $\preccurlyeq$ be a relation is defined as a set of ordered pairs such that $x \preccurlyeq y(x, y \in \mathbb{R})$. This relation may be regarded as a transversal upper or lower set $A$ in $\mathbb{R} \times \mathbb{R}$. Then for $g($ or $d): I \rightarrow I$ defined by $g(x)=1-x$ for $x \in[0,1 / 2]$ and $g(x)=1 / 3$ for $x \in(1 / 2,1]$, and for $f_{A}(5,10)=0, f_{A}(10,99)=0.5, f_{A}(11,100)=1$, etc., we obtain a characterization of the binary transversal upper or lower relation $A$ in $X \times X$, where $f_{A}(x, y): X \times X \rightarrow I$.

\section{Minimax FACTS ON TRANSVERSAL SETS}

This section gives applications of the preceding objects of transversal upper or lower sets.

In connection with the preceding, the following our former results allows us to prove the basic statements for further facts.

Let $(P, \preccurlyeq)$ be a partially ordered set by the ordering relation $\preccurlyeq$. The function $g: P^{k} \rightarrow P$ ( $k$ is a fixed positive integer) is decreasing on the ordered set $P$ if $a_{i}, b_{i} \in P$ and $a_{i} \preccurlyeq b_{i}(i=1, \ldots, k)$ implies $g\left(b_{1}, \ldots, b_{k}\right) \preccurlyeq g\left(a_{1}, \ldots, a_{k}\right)$.

Let $L$ be a lattice and $g$ a mapping from $L^{k}$ into $L$. For any $g: L^{k} \rightarrow L$ it is natural to consider the following property of local comparability, which means, if $J \in L$ is comparable with $g(J, \ldots, J) \in L$ then $J$ is comparable with every $t \in L$.

We begin with the following essential statements from Taskovic [7].

Lemma 1. (Sup-Inf Inequalities). Let $(L, \preccurlyeq)$ be a lattice and let $g: L^{2} \rightarrow L$ be a decreasing mapping. If $L$ has the property of local comparability, then for arbitrary functions $p: X \rightarrow L$ and $q: Y \rightarrow L$ ( $X$ and $Y$ are arbitrary nonempty sets) the following relations are valid:

$$
\xi \preccurlyeq g(\xi, \xi) \quad \text { implies } \quad \xi \preccurlyeq \sup \{p(x), q(y), g(p(x), q(y))\},
$$

and

$$
g(\xi, \xi) \preccurlyeq \xi \quad \text { implies } \quad \inf \{p(x), q(y), g(p(x), q(y))\} \preccurlyeq \xi,
$$


for all $x \in X$ and for all $y \in Y$. Hence, in particular, $\xi=g(\xi, \xi)$ implies

$$
\inf \{p(x), q(y), g(p(x), q(y))\} \preccurlyeq \xi \preccurlyeq \sup \{p(x), q(y), g(p(x), q(y))\},
$$

for all $x \in X$ and for all $y \in Y$.

A brief proof of this statement based on the former facts may be found in Tasković [7].

An immediate consequence (special case for totally ordered sets) of the preceding Lemma 1 is the following its form.

Lemma 1a. (Minimax Inequalities). Let $P$ be a totally ordered set by the order relation $\preccurlyeq$, and let $g: P^{2} \rightarrow P$ be a decreasing mapping. Then for functions $p: X \rightarrow P$ and $q: Y \rightarrow P$ ( $X$ and $Y$ are nonempty sets) the following relations are valid:

$$
\xi \preccurlyeq g(\xi, \xi) \quad \text { implies } \quad \xi \preccurlyeq \max \{p(x), q(y), g(p(x), q(y))\},
$$

and

$$
g(\xi, \xi) \preccurlyeq \xi \quad \text { implies } \quad \min \{p(r), q(s), g(p(r), q(s))\} \preccurlyeq \xi,
$$

for all $x, r \in X$ and for all $y, s \in Y$. Hence, in particular, $\xi=g(\xi, \xi)$ implies

$$
\min \{p(r), q(s), g(p(r), q(s))\} \preccurlyeq \xi \preccurlyeq \max \{p(x), q(y), g(p(x), q(y))\},
$$

for all $x, r \in X$ and for all $y, s \in Y$.

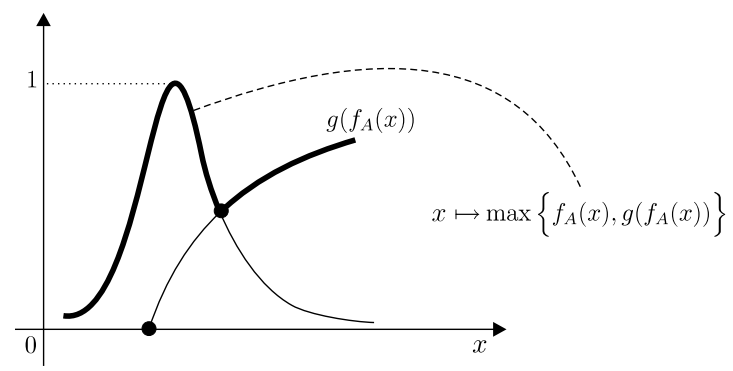

Figure 6

We notice, quantifying the assertions (S), (I) and (U) we obtain the following interesting conclusions (which, incidentally are their equivivalent formulations for $X=Y)$ :

$$
\xi \preccurlyeq g(\xi, \xi) \quad \text { implies } \quad \xi \preccurlyeq \inf _{x, y \in X} \sup \{p(x), q(y), g(p(x), q(y))\},
$$

and

$$
g(\xi, \xi) \preccurlyeq \xi \quad \text { implies } \quad \sup _{x, y \in X} \inf \{p(x), q(y), g(p(x), q(y))\} \preccurlyeq \xi ;
$$

and $g(\xi, \xi)=\xi$ implies the following inequalities:

(EU)

$$
\sup _{x, y \in X} \inf \{p(x), q(y), g(p(x), q(y))\} \preccurlyeq \xi \preccurlyeq \inf _{x, y \in X} \sup \{p(x), q(y), g(p(x), q(y))\} .
$$


Remark. The above statements (Lemma 1) still hold when $g: L^{k} \rightarrow L$ ( $k$ is a fixed positive integer) is a decreasing function. The proof is quite similar; the assertions corresponding to (S) and (I) look as follows:

$$
\xi \preccurlyeq g(\xi, \ldots, \xi) \text { implies } \xi \preccurlyeq \sup \left\{\lambda_{1}, \ldots, \lambda_{k}, g\left(\lambda_{1}, \ldots, \lambda_{k}\right)\right\}
$$

and

$$
g(\xi, \ldots, \xi) \preccurlyeq \xi \quad \text { implies } \inf \left\{\lambda_{1}, \ldots, \lambda_{k}, g\left(\lambda_{1}, \ldots, \lambda_{k}\right)\right\} \preccurlyeq \xi
$$

for arbitrary functions $\lambda_{1}, \ldots, \lambda_{k}: X \rightarrow L$, where $X$ is an arbitrary nonempty set. Also, in particular, $\xi=g(\xi, \ldots, \xi)$ implies

$$
\inf \left\{\lambda_{1}, \ldots, \lambda_{k}, g\left(\lambda_{1}, \ldots, \lambda_{k}\right)\right\} \preccurlyeq \xi \preccurlyeq \sup \left\{\lambda_{1}, \ldots, \lambda_{k}, g\left(\lambda_{1}, \ldots, \lambda_{k}\right)\right\}
$$

for arbitrary functions $\lambda_{i}: X \rightarrow L(i=1, \ldots, k)$, where $X$ is an arbitrary nonempty set. To simplify the notation we will give the proof only for the case $k=2$.

On the other hand, we note, that it is easy to construct a decreasing mapping on a complete lattice vhich is not a totally ordered set but the property of local comparability is fulfilled, see Figure 7.

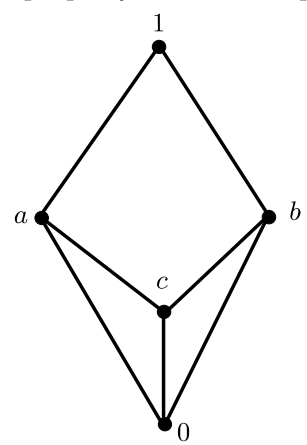

Figure 7

Example 3. Let $L$ be the lattice on Figure 7 and let $g: L \rightarrow L$ be defined by $g(0)=1$, $g(a)=b, g(b)=a, g(c)=0, g(1)=0$. Evidently, $g$ is decreasing and the property of local somparability is fulfilled, but the set $L$ is not totally ordered.

Proof of Lemma 1. Implication (S). Let $\zeta \preccurlyeq g(\zeta, \zeta)$ and $\lambda=\sup \{p(x), q(y)\}$, where the elements $x \in X$ and $y \in Y$ are arbitrarily chosen. If $\zeta \preccurlyeq \lambda$ then

$$
\zeta \preccurlyeq \sup \{p(x), q(y), g(p(x), q(y))\}
$$

for all $x \in X$ and $y \in Y$, obviously holds. If $\lambda \preccurlyeq \zeta$, then $\zeta \preccurlyeq g(\zeta, \zeta) \preccurlyeq g(p(x), q(y))$ and (6) holds too. We see that the comparability of elements $\lambda$ and $\zeta$ is possible as a consequence of the property of local comparability.

One gets the implication (I) by applying the above results to the case where the relation $\preccurlyeq$ is replaced by the relation $\succcurlyeq$; in fact, after this change, every supremmum becomes an infimum and the function $g$ remains decreasing with respect to each argument. Thus, we have (I). The last assertion (U) is evident. Thus, the proof is complete.

Lemma 2. (Tasković, [7]). Let $P$ be a totally ordered set by the order relation $\preccurlyeq$, and let $g: L^{2} \rightarrow L$ be a decreasing mapping. Then, the following conditions are equivalent:

$$
\min \{t, g(t, t)\} \preccurlyeq \zeta \preccurlyeq \max \{t, g(t, t)\}
$$

for all $t \in P$, and the following condition

$$
\zeta=\min P_{g} \text { or } \zeta=\max P^{g}
$$

where in the preceding $P_{g}:=\{t \in P \mid g(t, t) \preccurlyeq t\}$ and $P^{g}:=\{t \in P \mid t \preccurlyeq g(t, t)\}$. 
From this assertion as a direct consequence it follows that:

1) The number of points $\zeta \in P$ with characteristic (7) can be 0, 1 or 2.

2) Each of these cases can be realized.

3) If $P$ is an everywhere dense set of points, the number of points with characteristic (7) is 0 or 1.

4) If the set $P$ has the characteristic of density (:= that is for every Dedekind's cross section the lower class has the maximum or the upper class has a minimum) the number of points is 1 or 2.

5) If $\zeta \in P$ is the fixed point of the mapping $g: P^{2} \rightarrow P$ in this sense $g(\zeta, \zeta)=\zeta$, then $\zeta$ is the point with characteristic (7) and then (7) holds if and only if

$$
\max _{x \in P} \min \{x, g(x, x)\}=\min _{x \in P} \max \{x, g(x, x)\}:=\zeta .
$$
$[7]$.

A brief proof of the preceding statement based on the preceding facts may be founded in Tasko vić

Remark. In Lemma 2 the assumption that $(P, \preccurlyeq)$ is totally ordered cannot be replaced by the weaker assumption that $(P, \preccurlyeq)$ is a lattice. More preciesely, the implication $(7)$ implies $(8)$ holds true in the case of any poset, while the implication (8) implies (7) is in general false even for lattices. Indeed, from (7) it follows that each element $t \in P$ is comparable with $g(t, t)$ so that $\zeta \in P_{g}$ or $\zeta \in P^{g}$. In the first case $t \in P_{g}$, i.e., $g(t, t) \preccurlyeq t$; so we have $\zeta \preccurlyeq \max \{t, g(t, t)\}=t$, and hence $\zeta=\min P_{g}$. A symmetric proof shows that $\zeta \in P^{g}$ implies $\zeta=\max P^{g}$. On the other hand, the structure on Figure 8 is obviously a lattice and the function $g: P \rightarrow P$ defined by $g(a)=c, g(b)=g(d)=b, g(c)=a$, where $P=\{a, b, c, d\}$, is decreasing. In this case we have also $P_{g}=\{b, c\}, P^{g}=\{a, b\}$ and thus $b=\min P_{g}=\max P^{g}$, i.e., (8) holds. However (7) is false since $d$ is not comparable with $b=g(d)$.

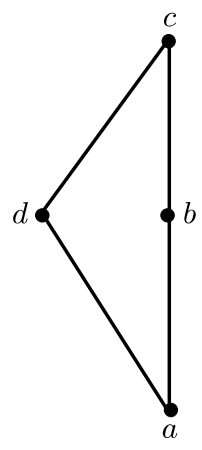

Figure 8

With the help of the preceding statements we now obtain the fundamental fact of tihis section.

Theorem 1 (Sup-Inf Theorem). Let $(L, \preccurlyeq)$ be a lattice and let $g: L^{2} \rightarrow L$ be a decreasing mapping. If $L$ has the property of local comparability, then for some arbitrary functions $p: X \rightarrow L$ and $q: Y \rightarrow L$ ( $X$ and $Y$ are arbitrary nonempty sets) the equality

(SI)

$$
\max _{x \in X, y \in Y} \inf \{p(x), q(y), g(p(x), q(y))\}=\min _{x \in X, y \in Y} \sup \{p(x), q(y), g(p(x), q(y))\}
$$

holds if and only if

$$
\inf \left\{p\left(x_{0}\right), q\left(y_{0}\right), g\left(p\left(x_{0}\right), q\left(y_{0}\right)\right)\right\}=\sup \left\{p\left(r_{0}\right), q\left(z_{0}\right), g\left(p\left(r_{0}\right), q\left(z_{0}\right)\right)\right\}
$$


for some $x_{0}, r_{0}, \in X$ and some $y_{0}, z_{0} \in Y$.

Proof. This follows at once from (EU) of Lemma 1 and the trivial fact that the strict inequality cannot hold in (EU).

In this sense, the necessity of the condition being trivial, we only prove its sufficiency. If $(\mathrm{Si})$ holds, then we have the following relations

$$
p\left(r_{0}\right), q\left(z_{0}\right), g\left(p\left(r_{0}\right), q\left(z_{0}\right)\right) \preccurlyeq s=i \preccurlyeq p\left(x_{0}\right), q\left(y_{0}\right), g\left(p\left(x_{0}\right), q\left(y_{0}\right)\right)
$$

for $s:=\sup \left\{p\left(r_{0}\right), q\left(z_{0}\right), g\left(p\left(r_{0}\right), q\left(z_{0}\right)\right)\right\}, i:=\inf \left\{p\left(x_{0}\right), q\left(y_{0}\right), g\left(p\left(x_{0}\right), q\left(y_{0}\right)\right)\right\}$, and for some $x_{0}, r_{0} \in X$ and $y_{0}, r_{0} \in Y$. Since $g: L^{2} \rightarrow L$ is decreasing, from the facts (9) we obtain

$$
g(i, i)=g(s, s) \preccurlyeq g\left(p\left(r_{0}\right), q\left(z_{0}\right)\right) \preccurlyeq s=i \preccurlyeq g\left(p\left(x_{0}\right), q\left(y_{0}\right)\right) \preccurlyeq g(s, s)=g(i, i),
$$

i.e., $i=s=g(i, i)=g(s, s)$. Applying Lemma 1 (case (U)) from the local comparability we have

$$
\inf \{p(x), q(y), g(p(x), q(y))\} \preccurlyeq i=s \preccurlyeq \sup \{p(x), q(y), g(p(x), q(y))\}
$$

for all $x \in X$ and $y \in Y$. Therefore, we have (SI). The proof is complete.

An immediate consequence (special case) of the preceding statement is the following principle.

Theorem 1a (Minimax Principle). Let $P$ be a totally ordered set by the order relation $\preccurlyeq$, and let $g: P^{2} \rightarrow P$ be a decreasing mapping. Then for some arbitrary functions $p: X \rightarrow P$ and $q: Y \rightarrow P(X$ and $Y$ are arbitrary nonempty sets) the equality

(MM) $\max _{x \in X, y \in Y} \min \{p(x), q(y), g(p(x), q(y))\}=\min _{x \in X, y \in Y} \max \{p(x), q(y), g(p(x), q(y))\}$

holds if and only if

$$
p\left(x_{0}\right)=q\left(y_{0}\right):=\xi=g(\xi, \xi) \quad \text { for some } x_{0} \in X \text { and } y_{0} \in Y .
$$

Proof. Applying Theorem 1 we obtain that (MM) is an equivalent with ( $\mathrm{Si}$ ), i.e., since $P$ is a totally ordered set the equality $(\mathrm{Si})$ is in the following form

$$
\min \left\{p\left(x_{0}\right), q\left(y_{0}\right), g\left(p\left(x_{0}\right), q\left(y_{0}\right)\right)\right\}=\max \left\{p\left(r_{0}\right), q\left(z_{0}\right), g\left(p\left(r_{0}\right), q\left(z_{0}\right)\right)\right\}
$$

for some totally comparable elements $p\left(x_{0}\right), q\left(y_{0}\right), g\left(p\left(x_{0}\right), q\left(y_{0}\right)\right), p\left(r_{0}\right), q\left(z_{0}\right)$ and the element $g\left(p\left(r_{0}\right)\right.$, $\left.q\left(z_{0}\right)\right)$ on $P$. Hence, we get that $p\left(x_{0}\right)=q\left(y_{0}\right)=p\left(r_{0}\right)=q\left(z_{0}\right)$, i.e., from $\left(9^{\prime}\right)$ we have $\xi:=p\left(x_{0}\right)=$ $q\left(y_{0}\right)=g(\xi, \xi)$, i.e., (Mm). The proof is complete.

The statement above still holds when $g: P^{k} \rightarrow P$ ( $k$ is a fixed positive integer) is a decreasing function. The proof is quite similar. Therefore, let $(P, \preccurlyeq)$ be a totally ordered set by the order relation $\preccurlyeq$, and $g: P^{k} \rightarrow P$ ( $k \in \mathbb{N}$ is fixed) be a decreasing mapping. Then, the equality

$$
\begin{aligned}
& \max _{\lambda_{1}, \ldots, \lambda_{k} \in P} \min \left\{\lambda_{1}, \ldots, \lambda_{k}, g\left(\lambda_{1}, \ldots, \lambda_{k}\right)\right\}= \\
& =\min _{\lambda_{1}, \ldots, \lambda_{k} \in P} \max \left\{\lambda_{1}, \ldots, \lambda_{k}, g\left(\lambda_{1}, \ldots, \lambda_{k}\right)\right\}
\end{aligned}
$$


holds if and only if

$$
\lambda_{1}\left(a_{1}\right)=\cdots=\lambda_{k}\left(a_{k}\right):=\xi=g(\xi, \ldots, \xi) \text { for some } a_{1}, \ldots, a_{k} \in X,
$$

where $\lambda_{i}: X \rightarrow P(i=1, \ldots, k)$ are arbitrary functions and $X$ is a nonempty set.

We remark that when $X=P, p(x)=x$ and $q(y)=y$ Theorem 1a reduces to that of our the following former result.

Corollary 1 (Tasković, [7]). Let $P$ be a totally ordered set by the order relation $\preccurlyeq$, and let $g: P^{2} \rightarrow P$ be a decreasing mapping. Then the equality

$$
\max _{x, y \in P} \min \{x, y, g(x, y)\}=\min _{x, y \in P} \max \{x, y, g(x, y)\}
$$

holds if and only if there is $\xi \in P$ such that $g(\xi, \xi)=\xi$.

In connection with the preceding, we note that we can give an extension of the preceding Theorem 1, as a direct consequence of the preceding facts, in the following sense.

Theorem 2 (General Sup-Inf Theorem)). Let $(L, \preccurlyeq)$ be a lattice and let $g: L^{2} \rightarrow$ $L$ be a mapping. Then for some arbitrary $p: X \rightarrow L$ and $q: Y \rightarrow L(X$ and $Y$ are arbitrary nonemptu sets) the following equality holds

$$
\max _{x \in X, y \in Y} \inf \{p(x), q(y), g(p(x), q(y))\}=\min _{x \in X, y \in Y} \sup \{p(x), q(y), g(p(x), q(y))\}
$$

if and only if the following inequalities hold

$$
\begin{aligned}
& \inf \{p(x), q(y), g(p(x), q(y))\} \preccurlyeq \inf \left\{p\left(x_{0}\right), q\left(y_{0}\right), g\left(p\left(x_{0}\right), q\left(y_{0}\right)\right)\right\}= \\
& =\sup \left\{p\left(r_{0}\right), q\left(z_{0}\right), g\left(p\left(r_{0}\right), q\left(z_{0}\right)\right)\right\} \preccurlyeq \sup \{p(x), q(y), g(p(x), q(y))\}
\end{aligned}
$$

for some $x_{0}, y_{0} \in X$ and $r_{0}, z_{0} \in Y$, and for all $x \in X$ and $y \in Y$.

On the other hand, if $L$ is a totally ordered set and $g: L^{2} \rightarrow L$ a decreasing mapping, then condition (DI) is an equivalent with the following equality

$$
\max _{x \in X, y \in Y} \min \{p(x), q(y), g(p(x), q(y))\}=\min _{x \in X, y \in Y} \max \{p(x), q(y), g(p(x), q(y))\} .
$$

Also, in connection with the preceding equality (Uk), if $g: P^{k} \rightarrow P$ ( $k$ is a fixed positive integer) is not decreasing mapping, we can extension equality (Uk). In this sense, if $g: P^{k} \rightarrow P(k$ is a fixed positive integer) some arbitrary mapping then equality (Uk) holds if and only if the following inequalities hold

$$
\begin{aligned}
\min \left\{\lambda_{1}, \ldots, \lambda_{k}, g\left(\lambda_{1}, \ldots, \lambda_{k}\right)\right\} & \preccurlyeq \min \left\{\lambda_{1}\left(a_{1}\right), \ldots, \lambda_{k}\left(a_{k}\right), g\left(\lambda_{1}\left(a_{1}\right), \ldots, \lambda_{k}\left(a_{k}\right)\right)\right\}= \\
& =\max \left\{\lambda_{1}\left(b_{1}\right), \ldots, \lambda_{k}\left(b_{k}\right), g\left(\lambda_{1}\left(b_{1}\right), \ldots, \lambda_{k}\left(b_{k}\right)\right)\right\} \preccurlyeq \\
& \preccurlyeq \max \left\{\lambda_{1}, \ldots, \lambda_{k}, g\left(\lambda_{1}, \ldots, \lambda_{k}\right)\right\}
\end{aligned}
$$

for some $a_{1}, b_{1}, \ldots, a_{k}, b_{k} \in X$, where $\lambda_{i}: X \rightarrow P(i=1, \ldots, k)$ are arbitrary functions and $X$ is a nonempty set. 


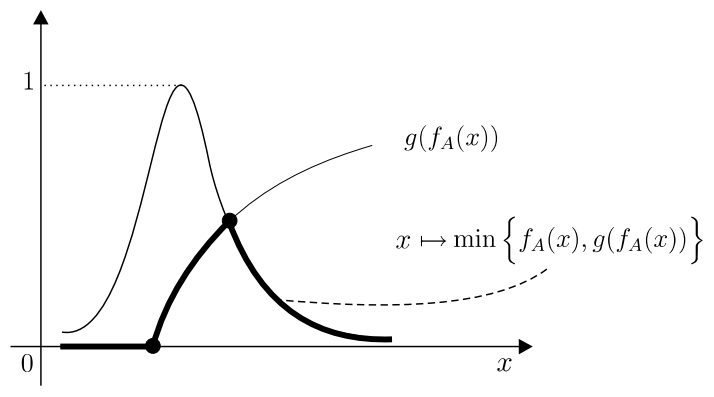

Figure 9

On the other hand, the next result follows from the preceding statements.

Corollary 2. Let $L$ be a lattice with the order relation $\preccurlyeq$. Then for some arbitrary mappings $p: X \rightarrow L$ and $q: X \rightarrow L$ ( $X$ is an arbitrary nonempty set) the following equality holds

$$
\max _{x, y \in X} \inf \{p(x), q(y)\}=\min _{x, y \in X} \sup \{p(x), q(y)\}
$$

if and only if the following inequalities hold

$$
\inf \{p(x), q(y)\} \preccurlyeq \inf \left\{p\left(x_{0}\right), q\left(y_{0}\right)\right\}=\sup \left\{p\left(r_{0}\right), q\left(z_{0}\right)\right\} \preccurlyeq \sup \{p(x), q(y)\}
$$

for some $x_{0}, y_{0}, r_{0}, z_{0} \in X$ and for all $x, y \in X$.

We note, in the preceding statements we can defined the preceding functions $p, q: X \rightarrow L$ and different sets, in sense that $p: X \rightarrow L$ and $q: Y \rightarrow L(X$ and $Y$ are arbitrary nonempty sets). Then the preceding statements hold too. In this sense, for some arbitrary functions $f_{i}: X_{i} \rightarrow L(i=1, \ldots, k)$ the following equality holds

$$
\max _{x_{1} \in X_{1}, \ldots, x_{k} \in X_{k}} \inf \left\{f_{1}\left(x_{1}\right), \ldots, f_{k}\left(x_{k}\right)\right\}=\min _{x_{1} \in X_{1}, \ldots, x_{k} \in X_{k}} \sup \left\{f_{1}\left(x_{1}\right), \ldots, f_{k}\left(x_{k}\right)\right\}
$$

if and only if the following inequalities hold

$$
\begin{aligned}
& \inf \left\{f_{1}\left(x_{1}\right), \ldots, f_{k}\left(x_{k}\right)\right\} \preccurlyeq \inf \left\{f_{1}\left(a_{1}\right), \ldots, f_{k}\left(a_{k}\right)\right\}= \\
& =\sup \left\{f_{1}\left(b_{1}\right), \ldots, f_{k}\left(b_{k}\right)\right\} \preccurlyeq\left\{f_{1}\left(x_{1}\right), \ldots, f_{k}\left(x_{k}\right)\right\}
\end{aligned}
$$

for some $a_{i}, b_{i} \in X_{i}(i=1, \ldots, k)$ and for all $x_{i} \in X_{i}(i=1, \ldots, k)$.

In this part of this section, we show that the existence of a separation in the preceding sense, is essential for applications of the preceding statements. This is a separation for the preceding equalities of the minimax type.

In this sense we give a characterization of a general variational equality. It is the following result.

Theorem 3 (Statement of Separation). Let $L$ be a lattice with the order relation $\preccurlyeq$, and with the local comparability. Then for some arbitrary mappings $p: X \rightarrow L$ and $q: Y \rightarrow L$ ( $X$ and $Y$ are two arbitrary nonempty sets) the following equality holds

$$
\operatorname{Max}_{x \in X} p(x)=\operatorname{Min}_{y \in Y} q(y)
$$


if and only if there exists a decreasing function $g: L^{2} \rightarrow L$ such that the following inequalities hold

$$
p(x) \preccurlyeq g(p(x), q(y)) \preccurlyeq q(y)
$$

for all $x \in X$ and $y \in Y$, and if there is $\xi \in L$ such that the $\xi \cap p(X)$ and $\xi \cap q(Y)$ are nonempty sets.

Proof. Necessity. Let the inequalities (PQ) hold and let, from the conditions, there exist points $x_{0} \in X$ and $y_{0} \in Y$ such that $\xi=p\left(x_{0}\right)=q\left(y_{0}\right)$. Thus, we obtain the following inequalities and equality of form (from Lemma 1)

$$
\inf \{p(x), q(y), g(p(x), q(y))\} \preccurlyeq \xi=g(\xi, \xi) \preccurlyeq \sup \{p(x), q(y), g(p(x), q(y))\}
$$

for some $x_{0} \in X$ and $y_{0} \in Y$, and for all $x \in X$ and $y \in Y$. This means, from Theorem 1 and from (PQ), that the equality (MM) holds, which give the equality (IS) of this statement.

Sufficiently. Assume that equality (IS) holds. Thus, there is $\xi \in L$ such that $p(x) \preccurlyeq \xi \preccurlyeq q(y)$ for all $x \in X$ and $y \in Y$, where $p\left(x_{0}\right)=q\left(y_{0}\right)=\xi$ for some $x_{0} \in X$ and $y_{0} \in Y$. If a decreasing function $g: L^{2} \rightarrow L$ defined by $g(s, t)=\xi$, then, directly, we obtain inequalities (PQ). The proof is complete.

In further, we give the following characterization a minimax equality via finite sets for transversal (upper or lower) sets.

Theorem 4. Let $P$ be a totally ordered set with the order relation $\preccurlyeq$ and let $g: P^{2} \rightarrow P$ be a mapping. Let $p: X \rightarrow P$ and $q: Y \rightarrow P(X$ and $Y$ are nonempty sets) such that $(x, y) \mapsto \max \{p(x), q(y), g(p(x), q(y))\}$ has a minimum on $X$ and $(x, y) \mapsto \min \{p(x), q(y), g(p(x), q(y))\}$ has a maximum on $Y$. Then the equality

$$
\begin{aligned}
& \left.\min _{x \in X} \max _{y \in Y} \max \{p(x), q(y), g(p(x), q(y)))\right\}= \\
& \left.=\max _{y \in Y} \min _{x \in X} \min \{p(x), q(y), g(p(x), q(y)))\right\}
\end{aligned}
$$

holds if and only if for any two finite sets $\left\{x_{1}, \ldots, x_{n}\right\} \subset X$ and $\left\{y_{1}, \ldots, y_{m}\right\} \subset Y$ there exist $x_{0} \in X$ and $y_{0} \in Y$ such that

$$
\max \left\{p\left(x_{0}\right), q\left(y_{k}\right), g\left(p\left(x_{0}\right), q\left(y_{k}\right)\right)\right\} \preccurlyeq \min \left\{p\left(x_{i}\right), q\left(y_{0}\right), g\left(p\left(x_{i}\right), q\left(y_{0}\right)\right)\right\}
$$

for $1 \leq i \leq n$ and $1 \leq k \leq m$.

Proof. Let the equality (10) holds. Then there exist $x_{0} \in X$ and $y_{0} \in Y$ such that the following inequalities hold

$$
\begin{aligned}
\max \left\{p\left(x_{0}\right), q\left(y_{k}\right), g\left(p\left(x_{0}\right), q\left(y_{k}\right)\right)\right\} & \preccurlyeq \max _{y \in Y} \max \left\{p\left(x_{0}\right), q(y), g\left(p\left(x_{0}\right), q(y)\right)\right\}= \\
& =\min _{x \in X} \min \left\{p(x), q\left(y_{0}\right), g\left(p(x), q\left(y_{0}\right)\right)\right\} \preccurlyeq \\
& \preccurlyeq \min \left\{p\left(x_{i}\right), q\left(y_{0}\right), g\left(p\left(x_{i}\right), q\left(y_{0}\right)\right)\right\}
\end{aligned}
$$

for all $i=1,2, \ldots, n$ and for all $k=1,2, \ldots, m$. This means that (11) holds. Conversely, according to this condition, from (11), we have that

$$
\max _{1 \leq k \leq m} \max \left\{p\left(x_{0}\right), q\left(y_{k}\right), g\left(p\left(x_{0}\right), q\left(y_{k}\right)\right)\right\} \preccurlyeq \min _{1 \leq i \leq n} \min \left\{p\left(x_{i}\right), q\left(y_{0}\right), g\left(p\left(x_{i}\right), q\left(y_{0}\right)\right)\right\}
$$

holds for any two finite sets $\left\{x_{1}, \ldots, x_{n}\right\} \subset X$ and $\left\{y_{1}, \ldots, y_{m}\right\} \subset Y$. Thus, we have

$$
\begin{aligned}
& \max _{m \leq \rho \leq \operatorname{Card} Y} \max _{1 \leq k \leq m} \max \left\{p\left(x_{0}\right), q\left(y_{k}\right), g\left(p\left(x_{0}\right), q\left(y_{k}\right)\right)\right\} \preccurlyeq \\
\preccurlyeq & \min _{n \leq \rho \leq \operatorname{Card} X} \min _{1 \leq i \leq n} \min \left\{p\left(x_{i}\right), q\left(y_{0}\right), g\left(p\left(x_{i}\right), q\left(y_{0}\right)\right)\right\},
\end{aligned}
$$


i. e., thus we obtain the following inequality

$$
\max _{y \in Y} \max \left\{p\left(x_{0}\right), q(y), g\left(p\left(x_{0}\right), q(y)\right)\right\} \preccurlyeq \min _{x \in X} \min \left\{p(x), q\left(y_{0}\right), g\left(p(x), q\left(y_{0}\right)\right)\right\},
$$

i.e., we obtain, from the conditions of statement, the following inequality

$$
\min _{x \in X} \max _{y \in Y} \max \{p(x), q(y), g(p(x), q(y))\} \preccurlyeq \max _{y \in Y} \min _{x \in X} \min \{p(x), q(y), g(p(x), q(y))\} .
$$

Since the reverse inequality of (10a) trivially holds, thus we obtain the equality (10). The proof is complete.

\section{NEW GAMES ON TRANSVERSAL SETS}

The game theory is a mathematical search for the optimal balance of conflicting interests, such as between two partners. As such, it is applicable to a wide variety of situations: social games, economic sompetetion between organizations, conflicts in nature, and so on. In former, the optimal strategies for both partners turn out to be described by saddle points in 1928 of John von Neumann.

In this section, the optimal strategies for both partners turn out to be described by the equalities (MM), (Uk) and (10), whose existence we established in Section 2.

Let $(P, \preccurlyeq)$ be a totally ordered set and let $g: P^{2} \rightarrow P$ be a decreasing mapping. We consider two players, $A$ and $B$. Players $A$ and $B$ have available sets of strategies $X \subset P$ and $Y \subset P$, respectively. Each point $x \in X$ and $y \in Y$ represents a possible choice by $A$ and $B$, respectively. If $A$ chooses $x$, and $B$ chooses $y$, then the function $(x, y) \mapsto \max \{x, y, g(x, y)\}$ represents the gain by $A$ and the function $(x, y) \mapsto \min \{x, y, g(x, y)\}$ represents the gain by $B$. The point $\xi \in P$ is called an optimal strategy if the following equality holds

$$
\xi:=\max _{x \in X, y \in Y} \min \{x, y, g(x, y)\}=\min _{x \in X, y \in Y} \max \{x, y, g(x, y)\} .
$$

In connection with this, we notice that the existence of the preceding optimal strategy is established in Theorem 1a and, specially, in Corollary 1; as shown in Figure 3.

In further, let $g: P^{k} \rightarrow P$ ( $k$ is a fixed positive integer) be a decreasing function and consider players $A_{1}, \ldots, A_{k}$ with sets of strategies $X_{1}, \ldots, X_{k}$ in $P$, respectively. Each point $\lambda\left(x_{1}\right) \in X_{1}, \ldots, \lambda\left(x_{k}\right) \in X_{k}$ for $x_{1}, \ldots, x_{k} \in X$ where $X$ is a nonempty set represents a possible choice by $A_{1}, \ldots, A_{k}$; respectively. The point $\xi \in P$ is called an $k$-optimal strategy, in this case, if the following equality holds

$$
\begin{aligned}
\xi & :=\max _{\lambda\left(x_{1}\right) \in X_{1}, \ldots, \lambda\left(x_{k}\right) \in X_{k}} \min \left\{\lambda\left(x_{1}\right), \ldots, \lambda\left(x_{k}\right), g\left(\lambda\left(x_{1}\right), \ldots, \lambda\left(x_{k}\right)\right)\right\}= \\
& =\min _{\lambda\left(x_{1}\right) \in X_{1}, \ldots, \lambda\left(x_{k}\right) \in X_{k}} \max \left\{\lambda\left(x_{1}\right), \ldots, \lambda\left(x_{k}\right), g\left(\lambda\left(x_{1}\right), \ldots, \lambda\left(x_{k}\right)\right)\right\} .
\end{aligned}
$$

In analogous with the preceding facts, the existence of the $k$-optimal strategy, in the preceding case, we established in Theorem 1a (the case (Uk)). 


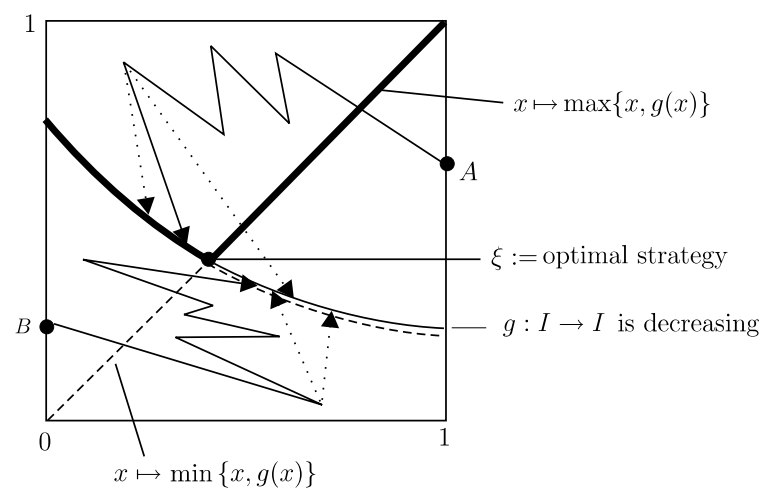

Figure 10

\section{EXISTENCE OF TRANSVERSAL POINTS}

In connection with the preceding, in this part we continue the study of the preceding minimax problems via transversal (upper or lower) sets. In this section we consider a concept of transversal points for the mapping $f$ of a nonempty set $X$ into partially ordered set $P$. A map $f$ of a nonempty set $X$ into partially ordered set $P$ has a transversal point $\zeta \in P$ if there is a decreasing function $g: P^{2} \rightarrow P$ such that the following equality holds

$$
\begin{aligned}
& \max _{x, y \in X} \min \{f(x), f(y), g(f(x), f(y))\}= \\
= & \min _{x, y \in X} \max \{f(x), f(y), g(f(x), f(y))\}:=\zeta .
\end{aligned}
$$

On the other hand, in our paper [10] we investigated the concept of fixed apices for a mapping $f$ of a set $X$ into itself. A map $f$ of a set $X$ to itself has a fixed apex $u \in X$ if for $u \in X$ there is $v \in X$ such that $f(u)=v$ and $f(v)=u$. The points $u, v \in X$ are called fixed apices of $f$ if $f(u)=v$ and $f(v)=u$. In this sense, a nonempty set $X$ is apices set if each of its points is an apex of some mapping $T: X \rightarrow X$. If $T: \mathbb{S}^{n} \rightarrow \mathbb{S}^{n}$ is the map such that $T x=-x$ for $x \in \mathbb{S}^{n}$, then $\mathbb{S}^{n}$ is an apices set.

Otherwise, a function $f: X \rightarrow P$ has a SI-transversal point if the preceding equality (12) holds with sup and inf instead max and min, respectively. If the preceding equality (12) holds for points $x,-x \in X$ ( $X$ is a linear space) $\zeta$ is A-transversal point; more generally $\zeta$ is R-transversal point for $f: X \rightarrow P$ if the equality (12) holds for points $x, T x \in X$. A function $f: X \rightarrow P(X$ is a linear space) has a pair of antipodal points $p,-p \in X$ if the following equality holds $f(p)=f(-p)$.

We note that from the second section, i.e., from Corollary 1 , we obtain that the function $f(x)=i d_{\mathbb{R}}$ : $\mathbb{R} \rightarrow \mathbb{R}$ has a transversal point $\zeta \in \mathbb{R}:=(-\infty,+\infty)$ if and only if for some decreasing function $g: \mathbb{R}^{2} \rightarrow \mathbb{R}$ we have $g(\zeta, \zeta)=\zeta$.

Let $E$ be the normed space of all those sequences $x=\left(x_{1}, x_{2}, \ldots\right)$ of real numbers having at most finitely many $x_{n} \neq 0$, with the norm $\|x\|=\sum\left|x_{i}\right|$. The subset $\left\{x \in E \mid x_{i}=0\right.$ for all $\left.i>n\right\}$ is denoted by $E^{n}$ or $\mathbb{R}^{n}$; the unit $n$-ball is $V^{n}=\left\{x \in E^{n}:\|x\| \leq 1\right\}$. The unit $n$-sphere $\mathbb{S}^{n}=\left\{x \in E^{n+1}:\|x\|=1\right\}$; 
its upper hemisphere is $\mathbb{S}_{+}^{n}=\left\{x \in \mathbb{S}^{n}: x_{n+1} \geq 0\right\}$, and its lower hemisphere is $\mathbb{S}_{-}^{n}=\left\{x \in \mathbb{S}^{n}: x_{n+1} \leq 0\right\}$; clearly $\mathbb{S}^{n}=\mathbb{S}_{+}^{n} \cup \mathbb{S}_{-}^{n}$. Observe that for any $k<n$, we have

$$
\mathbb{S}^{k}=\left\{x \in \mathbb{S}^{n} \mid x_{k+2}=\cdots=x_{n+1}=0\right\}
$$

and that $\mathbb{S}^{n-1}=\mathbb{S}_{+}^{n} \cap \mathbb{S}_{-}^{n}$. Recall that a map $f: \mathbb{S}^{n} \rightarrow \mathbb{S}^{n}$ is antipodal-preserving if $f(\alpha)=\alpha(f)$ for some $\alpha: \mathbb{S}^{n} \rightarrow \mathbb{S}^{n}$.

Results equivalent to the Lusternik, Schnirelman and Borsuk statement use the notions of extendability and homotopy in their formulation. For the convenience of the reader, and to establish the terminology, we recall the relevant definitions. By space we understand a Hausdorff space; unless specifically stated otherwise, a map is a continuous transformation.

Let $X, Y$ be two spaces and $A \subset X$. A map $f: A \rightarrow Y$ is called extendable over $X$ if there is a map $F: X \rightarrow Y$ with $F \mid A=f$. Two maps $f, g: X \rightarrow Y$ are called homotopis if there is a map $H: X \times I \rightarrow Y$ with $H(x, 0)=f(x)$ and $H(x, 1)=g(x)$ for each $x \in X$. The map $H$ is called homotopy (or continuous deformation) of $f$ to $g$, and written $H: f \cong g$. For each $t$, the map $x \mapsto H(x, t)$ is denoted by $H_{t}: X \rightarrow Y$ clearly the family $\left(H_{t}\right)_{0 \leq t \leq 1}$ determines $H$ and vice versa. Thus, the realtion of homotopy decomposes the set of all maps of $X$ into $Y$ into pairwise disjoint classes called homotopy classes and $f: X \rightarrow Y$ homotopis to a constant map is called nullhomotopic.

We can now prove Borsuk's antipodal theorem and also show that it is equivalent to various geometric results about the $n$-sphere.

Theorem 5 (Tasković, $[10]$ ). Let $\mathbb{S}^{n}$ denote the $n$-sphere. Then the following statements are equivalent:

(a) (Lusternik-Schnirelman-Borsuk theorem). In any closed covering with $\left\{M_{1}, \ldots, M_{n+1}\right\}$ of $\mathbb{S}^{n}$ by $(n+1)$-sets, at least one set of the following form $M_{i}(i=1, \ldots, n+1)$ must contain a pair of antipodal points.

(b) (Borsuk antipodal theorem). An antipodal-preserving map $f: \mathbb{S}^{n-1} \rightarrow$ $\mathbb{S}^{n-1}$ is not nullhomotopic.

(c) (Borsuk-Ulam type theorem). Every continuous map $f: \mathbb{S}^{n} \rightarrow \mathbb{R}$ sends at least one pair of antipodal points to the same point.

(d) Every continuous map $f: \mathbb{S}^{n} \rightarrow \mathbb{R}$ has at least one A-transversal point.

A brief proof of this statement based on the former facts may be found in Tasković [10].

In the connection with the former results of Lusternik, Schnirelman, Borsuk and Theorem 5, as an immediate consequence we obtain the following fact.

Corollary 3. Let $\mathbb{S}^{n}$ denotes the $n$-sphere. Then following statements are equivalent:

(a) (Borsuk-Ulam theorem). Every continuous map $f: \mathbb{S}^{n} \rightarrow \mathbb{R}^{n}$ sends at least one pair of antipodal points to the same point.

(b) (Tasković, [10]). Every continuous map $f: \mathbb{S}^{n} \rightarrow \mathbb{R}$ has at least one A-transversal point.

On the other hand, analogous to the preceding statement, we obtain the following extension of the former results.

Theorem 6. Let $X$ be an apices set in sense of fixed point free map $T: X \rightarrow X$ and let Card $X \geq$ continuum, then the following statements are equivalent: 
(a) In any closed covering $\left\{M_{1}, \ldots, M_{n+1}\right\}$ of $X$ by $(n+1)$-sets, at least one set $M_{i}(i=1, \ldots, n+1)$ must contain a pair of points $x, T x \in X$.

(b) Every continuous map $f: X \rightarrow \mathbb{R}$ has at least one pair of points of the form $p, T p \in X$ such that $f(p)=f(T p)$.

(c) Every continuous map $f: X \rightarrow \mathbb{R}$ has at least one $R$-transversal point.

The proof is analogous to the proof of the preceding Theorem 5 (see: Tasković $[10])$.

In connection with the transversal points, in this part we consider some other concepts of points for the mapping $f$ of a nonempty set $X$ into a partially ordered set $P$. A map $f: X \rightarrow P$ has a furcate point $\zeta \in P$ if for some function $T: X \rightarrow X$ the following equality holds

$$
\max _{x, y \in X} \min \{f(x), f(T y)\}=\min _{x, y \in X} \max \{f(x), f(T y)\}:=\zeta .
$$

Otherwise, a function $f: X \rightarrow P$ has a SI-furcate point if the preceding equality (13) holds when instead max and min stand sup and inf, respectively. If the preceding equality (13) holds for points $x,-x \in X$ ( $X$ is a linear space), then $\zeta$ is A-furcate point; or generally $\zeta$ is R-furcate point for $f: X \rightarrow P$ if the equality (13) holds for points $x, T x \in X$.

From the second section, i.e., from Theorem 2, we obtain that for the function $f: X \rightarrow L$ ( $X$ is an arbitrary nonempty set and $(L, \preccurlyeq)$ is a lattice) the following inequalities hold

$$
\inf \{f(x), f(T y)\} \preccurlyeq f\left(x_{0}\right)=f\left(T\left(y_{0}\right)\right) \preccurlyeq \sup \{f(x), f(T y)\}
$$

for some $x_{0}, y_{0} \in X$ and for all $x, y \in X$.

Thus, if $f: X \rightarrow L$ has an R-furcate point then $f$ has at least one pair of points $p, T p \in X$ such that $f(p)=f(T p)$. Reverse does not hold. Figure 11 shows the mapping $f$ of complete lattice $I:=[0,1]$ into itself with $f(p)=f(T p)$ for some $p \in I$, but without furcate points.

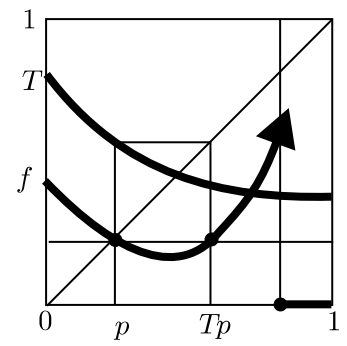

Figure 11

For two mappings $f: X \rightarrow P$ and $g: Y \rightarrow P(X$ and $Y$ are arbitrary nonempty sets and $P$ is a partially ordered set) we have common (coincidence) furcate points. Namely, two mappings $f: X \rightarrow P$ and $g: Y \rightarrow P$ have a coincidence furcate point $\zeta \in P$, if the following equality holds

$$
\max _{x \in X, y \in Y} \min \{f(x), g(y)\}=\min _{x \in X, y \in Y} \max \{f(x), g(y)\}:=\zeta .
$$


In general, the mappings $f_{i}: X_{i} \rightarrow P(i=1, \ldots, k)\left(X_{i}\right.$ are arbitrary nonempty sets and $P$ is a poset $:=$ partially ordered set) have a coincidence furcate point $\zeta \in P$ if the following equality holds

$$
\begin{aligned}
\max _{x_{1} \in X_{1}, \ldots, x_{k} \in X_{k}} \min \left\{f\left(x_{1}\right), \ldots, f\left(x_{k}\right)\right\} & = \\
= & \min _{x_{1} \in X_{1}, \ldots, x_{k} \in X_{k}} \max \left\{f\left(x_{1}\right), \ldots, f\left(x_{k}\right)\right\}:=\zeta .
\end{aligned}
$$

We notice that from Theorem 1a, we obtain that the functions $f: X \rightarrow P$ and $g: Y \rightarrow P$ have coincidence furcate point if and only if the following inequalities hold

$$
\min \{f(x), g(y)\} \preccurlyeq f\left(x_{0}\right)=g\left(y_{0}\right) \preccurlyeq \max \{f(x), g(y)\},
$$

for some $x_{0} \in X, y_{0} \in Y$ and for all $x \in X, y \in Y$. In connection with this, we notice that there are some continuous functions $f, g: I \rightarrow I$ (Figure 12) which map compact interval into itself, but $f$ and $g$ have not coincidence furcate points.

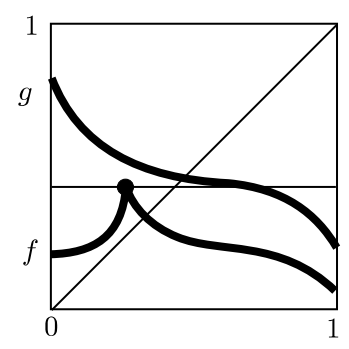

Figure 12

It is also possible to introduce the concept of general transversal point in the following sense: the function $f: X \rightarrow P$ ( $X$ is a nonempty set and $P$ a poset) has a quasi transversal point $\zeta \in P$, if for some function $g: P^{2} \rightarrow P$ the following equality holds

$$
\begin{aligned}
& \max _{x, y \in X} \min \{f(x), f(y), g(f(x), f(y))\}= \\
= & \min _{x, y \in X} \max \{f(x), f(y), g(f(x), f(y))\}:=\zeta .
\end{aligned}
$$

If in this equality $g(f(x), f(y))=r(x, y)$ then $\zeta$ is a TD-transversal point. We notice, from Theorem 1a, that mapping $f: X \rightarrow P$ has a general transversal point $\zeta \in P$ if and only if the following equality holds

$$
\begin{gathered}
\min \{f(x), f(y), g(f(x), f(y))\} \preccurlyeq f\left(x_{0}\right)=f\left(y_{0}\right):=\zeta= \\
=g(\zeta, \zeta) \preccurlyeq \max \{f(x), f(y), g(f(x), f(y))\},
\end{gathered}
$$

for some $x_{0}, y_{0} \in X$ and for all $x, y \in X$. Also, we can introduce SI-general transversal points, A-general points and R-general transversal points. 


\section{General transversal points}

In connection with former facts on transversal points, we have the following extensions. A map $f: X \rightarrow P$ ( $X$ is an arbitrary nonempty set and $P$ is a poset) has a general transversal point $\zeta \in P$ if there is a decreasing function $g: P^{k} \rightarrow P$ ( $k$ is a fixed positive integer) such that the following equality holds

$$
\begin{aligned}
& \max _{x_{1}, \ldots, x_{k} \in P} \min \left\{f\left(x_{1}\right), \ldots, f\left(x_{k}\right), g\left(f\left(x_{1}\right), \ldots, f\left(x_{k}\right)\right)\right\}= \\
= & \min _{x_{1}, \ldots, x_{k} \in P} \max \left\{f\left(x_{1}\right), \ldots, f\left(x_{k}\right), g\left(f\left(x_{1}\right), \ldots, f\left(x_{k}\right)\right)\right\}:=\zeta .
\end{aligned}
$$

From the second section, i.e., from Theorem 1a, case (Uk), we obtain that the function $f: X \rightarrow X$ has a general transversal point if and only if $f\left(t_{1}\right)=\cdots=$ $f\left(t_{k}\right):=\zeta=g(\zeta, \ldots, \zeta)$ for some $t_{1}, \ldots, t_{k} \in X$.

On the other hand, a map $f: X \rightarrow P(X$ is an arbitrary nonempty set and $P$ is a poset) has a quasi general transversal point $\zeta \in P$ if there is a function $g: P^{k} \rightarrow P$ ( $k$ is a fixed positive integer) such that the equality (14) holds. Also, from the former results, we obtain that the function $f: X \rightarrow P$ has a quasi general transversal point if and only if the following holds

$$
\begin{aligned}
& \min \left\{f\left(x_{1}\right), \ldots, f\left(x_{k}\right), g\left(f\left(x_{1}\right), \ldots, f\left(x_{k}\right)\right)\right\} \preccurlyeq f\left(t_{1}\right)=\cdots=f\left(t_{k}\right)=\zeta= \\
& =g(\zeta, \ldots, \zeta) \preccurlyeq \max \left\{f\left(x_{1}\right), \ldots, f\left(x_{k}\right), g\left(f\left(x_{1}\right), \ldots, f\left(x_{k}\right)\right)\right\}
\end{aligned}
$$

for some $t_{1}, \ldots, t_{k} \in X$ and for all $x_{1}, \ldots, x_{k} \in X$.

If the function $g: P^{k} \rightarrow P$ ( $k$ is a positive integer) is a decreasing function with the property $f\left(t_{1}\right)=\cdots=f\left(t_{k}\right):=\zeta=g(\zeta, \ldots, \zeta)$ for some $t_{1}, \ldots, t_{k} \in X$, then, from Theorem 1a (case (Uk)) and Lemma 1a, we obtain that the preceding condition (15) holds.

\section{Roots of ALGEBRAiC EQUATIONS AS TRANSVERSAL SETS}

We note that, by the application of Lemma 1a (in fact of (U), i.e., (Uk)) one can simultaneously obtain the upper and lower bounds of the roots of the following equation

$$
\begin{gathered}
x^{n}=a_{1} x^{n-1}+a_{2} x^{n-2}+\cdots+a_{n} \\
\left(a_{1}+\cdots+a_{n}>0 ; a_{i} \geq 0(i=1, \ldots, n)\right) .
\end{gathered}
$$

As an immediate consequence of Theorem 1a, the case (Uk), we obtain the following statement. 
Theorem 7. A point $\xi \in \mathbb{R}_{+}:=(0,+\infty)$ is the root of the equation $(A E)$ if and only if the following equality holds

$$
\begin{aligned}
\xi & :=\max _{\lambda_{2}, \ldots, \lambda_{n} \in \mathbb{R}_{+}} \min \left\{\lambda_{2}, \ldots, \lambda_{n}, a_{1}+\frac{a_{2}}{\lambda_{2}}+\cdots+\frac{a_{n}}{\lambda_{n}^{n-1}}\right\}= \\
& =\min _{\lambda_{2}, \ldots, \lambda_{n} \in \mathbb{R}_{+}} \max \left\{\lambda_{2}, \ldots, \lambda_{n}, a_{1}+\frac{a_{2}}{\lambda_{2}}+\cdots+\frac{a_{n}}{\lambda_{n}^{n-1}}\right\} .
\end{aligned}
$$

Remark 1. In connection with the preceding facts about transversal points, from Theorem 7, we obtain that the equation (AE) has a root $\zeta \in \mathbb{R}_{+}$if and only if the point $\zeta$ is a general transversal point of the function $f(x)=i d_{\mathbb{R}_{+}}: \mathbb{R}_{+} \rightarrow \mathbb{R}_{+}$.

Proof of Theorem 7. From Theorem 1a, the case (Uk), we may choose the decreasing function $g: P^{n} \rightarrow P, n \in \mathbb{N}$ is fixed, for $P:=\mathbb{R}_{+}$defined by

$$
g\left(x_{1}, \ldots, x_{n}\right)=a_{1}+\frac{a_{2}}{x_{2}}+\cdots+\frac{a_{n}}{x_{n}^{n-1}} \quad \text { for } x_{1}, \ldots, x_{n} \in \mathbb{R}_{+} .
$$

Applying, Theorem 1a, the case (Uk), we obtain directly the preceding equality (Ro) for the positive root of the equation (AE).

Remark 2. We notice that, if $0<a_{1}+\cdots+a_{n}<1$, then a root $\xi$ of the equation (AE) of the form (Ro) lie in the open interval $(0,1)$, i.e., $\xi \in(0,1)$, such that

$$
\begin{aligned}
\xi & =\max _{\lambda_{2}, \ldots, \lambda_{n} \in(0,1)} \min \left\{\lambda_{2}, \ldots, \lambda_{n}, a_{1}+\frac{a_{2}}{\lambda_{2}}+\cdots+\frac{a_{n}}{\lambda_{n}^{n-1}}\right\}= \\
& =\min _{\lambda_{2}, \ldots, \lambda_{n} \in(0,1)} \max \left\{\lambda_{2}, \ldots, \lambda_{n}, a_{1}+\frac{a_{2}}{\lambda_{2}}+\cdots+\frac{a_{n}}{\lambda_{n}^{n-1}}\right\} .
\end{aligned}
$$

Applying Theorem 1a, case (Uk), we obtain directly the following equality for positive root of equation (AE).

Theorem 8. Let $I_{1}, \ldots, I_{n}$ be indices sets and $\theta_{i_{j}} \geq 0$ be real numbers which satisfy the following condition

$$
\sum_{i_{j} \in I_{j}} \theta_{i_{j}}=j-t \quad \text { for } j=1, \ldots, n \text { and } 0<t<1 .
$$

Then $\zeta \in \mathbb{R}_{+}$is the root of the algebraic equation $x^{t}=a_{1} x^{t-1}+\cdots+a_{n} x^{t-n}$ $\left(\left(a_{1}, \ldots, a_{n}\right) \neq(0, \ldots, 0)\right)$ if and only if the following equality holds

$$
\begin{aligned}
& \max _{M_{i_{j}}} \min \left\{M_{i_{j}},\left(\sum_{j=1}^{n} \frac{a_{j}}{\prod_{i_{j} \in I_{j}} M_{i_{j}}^{\theta_{i_{j}}}}\right)^{1 / t}\right\}= \\
= & \min _{M_{i_{j}} \max }\left\{M_{i_{j}},\left(\sum_{j=1}^{n} \frac{a_{j}}{\prod_{i_{j} \in I_{j}} M_{i_{j}}^{\theta_{i_{j}}}}\right)^{1 / t}\right\}:=\zeta .
\end{aligned}
$$


Proof. In order to prove this statement we may choose the function $g: \mathbb{R}_{+}^{n} \rightarrow \mathbb{R}_{+}$ ( $n$ is a positive integer) defined by

$$
g\left(x_{1}, \ldots, x_{n}\right)=\left(\sum_{j=1}^{n} \frac{a_{j}}{\prod_{i_{j} \in I_{j}} x_{i_{j}}^{\theta_{i_{j}}}}\right)^{1 / t} \text { for } x_{1}, \ldots, x_{n} \in \mathbb{R}_{+}
$$

and then apply Theorem 1a case (Uk). With this the proof is complete.

\section{Transversal BCC-IDEals in BCC-Algebras}

In 1976, K. Iséki and S. Tanaka obtained some essential results in ideal theory of BCK-algebras.

In this section a binary multiplication will be denoted by juxtaposition. Dots we use only to avoid repetitions of brackets. For example, the formula $((x y)(z y))(x z)=$ $\boldsymbol{O}$ will be written as $(x y * z y) * x z=\boldsymbol{O}$.

A nonempty set $X$ with a constant $\boldsymbol{O}$ and a binary operation denoted by juxtaposition is called a BCC-algebra if for all $x, y, z \in X$ the following axioms hold: $(x y * z y) * x z=\boldsymbol{O}, x x=\boldsymbol{O}, \boldsymbol{O} x=\boldsymbol{O}, x \boldsymbol{O}=x, x y=\boldsymbol{O}$ and $y x=\boldsymbol{O}$ imply $x=y$.

Any BCK-algebra is a BCC-algebra. But, we notice that a BCC-algebra is a $\mathrm{BCK}$-algebra if and only if it satisfies $x y * z=x z * y$.

On any BCC-algebra (similarly as in the case of BCK-algebras) one can define the natural order $\preccurlyeq$ by putting $x \preccurlyeq y$ if and only if $x y=\boldsymbol{O}$. It is not difficult to verify that this order is partial and $\boldsymbol{O}$ is its smallest element.

A nonempty subset $A$ of $X$ is called a BCC-ideal of $X$ if $\boldsymbol{O} \in A$ and if $x y * z \in A$ and $y \in A$ imply $x z \in A$ for all $x, y, z \in X$.

In further, for the transversal upper or lower sets we consider a special case of the transversal membership function (1) or (4) if the lattice $L:=[a, b] \subset \mathbb{R}$ $(a<b ; a, b \in \mathbb{R})$.

Let $(P, \preccurlyeq)$ be a partially ordered set by the ordering relation $\preccurlyeq$. The function $g: P^{k} \rightarrow P$ ( $k$ is a fixed positive integer $)$ is increasing on the ordered set $P$ if $a_{i}, b_{i} \in P$ and $a_{i} \preccurlyeq b_{i}(i=1, \ldots, k)$ implies $g\left(a_{1}, \ldots, a_{k}\right) \preccurlyeq g\left(b_{1}, \ldots, b_{k}\right)$.

A transversal lower set $A$ in $X$ is called transversal lower BCC-ideal of $X$ if $f_{A}(\boldsymbol{O}) \geq f_{A}(x)$ and if there is a function $d:[a, b]^{2} \rightarrow[a, b]$ such that

$$
f_{A}(x z) \geq \min \left\{f_{A}(x y * z), f_{A}(y), d\left(f_{A}(x y * z), f_{A}(y)\right)\right\}
$$

for all $x, y, z \in X$. A transversal lower BCC-ideal $A$ of $X$ is said to be normal if there exists $x \in X$ such that $f_{A}(x)=b$.

We notice that if $A$ is a normal transversal lower BCC-ideal of $X$, then clearly $f_{A}(\boldsymbol{O})=b$, and hence $A$ is normal transversal lower BCC-ideal of $X$ if and only if $f_{A}(\boldsymbol{O})=b$.

We are now in a position to formulate the following statement for the transversal lower BCC-ideals.

Theorem 9. Given a transversal lower $B C C$-ideal $A$ of $X$ with the function $d$ in (Ld) which is increasing satisfying $d(t, t) \geq t$ for all $t \in[a, b]$ and let $B$ be $a$ 
transversal lower set in $X$ defined by $f_{B}(x)=f_{A}(x)+b-f_{A}(\mathbf{0})$ for all $x \in X$. Then $B$ is a normal transversal lower BCC-ideal of $X$ which contains $A$ with the function $d=r$ in $(L d)$ which is decreasing satisfying $r(t, t) \leq t$ for all $t \in[a, b]$.

Proof. For $x=\boldsymbol{O}$ we have $f_{B}(\boldsymbol{O})=f_{A}(\boldsymbol{O})+b-f_{A}(\boldsymbol{O})=b \geq f_{B}(x)$ for all $x \in X$. Further, let $x, y, z \in X$, then we obtain the following inequalities:

$$
\begin{gathered}
\min \left\{f_{B}(x y * z), f_{B}(y), r\left(f_{B}(x y * z), f_{B}(y)\right)\right\} \leq \\
\leq \min \left\{f_{B}(x y * z), f_{B}(y), r\left(\min \left\{f_{B}(x y * z), f_{B}(y)\right\}, \min \left\{f_{B}(x y * z), f_{B}(y)\right)\right\} \leq\right. \\
\left.\leq \min \left\{f_{B}(x y * z), f_{B}(y), \min \left\{f_{B}(x y * z), f_{B}(y)\right\}\right)\right\}= \\
\left.=\min \left\{f_{A}(x y * z)+b-f_{A}(\boldsymbol{O}), f_{A}(y)+b-f_{A}(\boldsymbol{O})\right)\right\}= \\
=\min \left\{f_{A}(x y * z), f_{A}(y)\right\}+b-f_{A}(\boldsymbol{O}) \leq \\
\leq \min \left\{f_{A}(x y * z), f_{A}(y), d\left(\min \left\{f_{A}(x y * z), f_{A}(y)\right\}, \min \left\{f_{A}(x y * z), f_{A}(y)\right\}\right)\right\}+ \\
+b-f_{A}(\boldsymbol{O}) \leq \min \left\{f_{A}(x y * z), f_{A}(y), d\left(f_{A}(x y * z), f_{A}(y)\right\}+b-f(\boldsymbol{O}) \leq\right. \\
\leq f_{A}(x z)+b-f_{A}(\boldsymbol{O})=f_{B}(x z),
\end{gathered}
$$

which shows that the set $B$ is a normal transversal lower BCC-ideal of $X$. Clearly $f_{A}(x) \leq f_{B}(x)$, completing the proof.

Theorem 10. Let $A$ be a transversal lower $B C C$-ideal of $X$ with the function $d$ in (Ld) which is increasing satisfying $d(t, t) \geq t$ for all $t \in[a, b]$ and let $\psi$ : $\left[\boldsymbol{O}, f_{A}(\boldsymbol{O})\right] \rightarrow[a, b]$ be an increasing function. Then a transversal lower set $B$ defined by $f_{\psi}(x):=\psi\left(f_{A}(x)\right)$ for all $x \in X$ is a transversal lower BCC-ideal of $X$ with the function $d=r$ in $(L d)$ which is decreasing satisfying $r(t, t) \leq t$ for all $t \in[a, b]$. Specially, if $\psi\left(f_{A}(\boldsymbol{O})\right)=b$, then $B$ is normal.

Proof. Since $\psi$ is an increasing function and $f_{A}(x) \leq f_{A}(\boldsymbol{O})$ for every $x \in X$, it follows that

$$
f_{\psi}(\boldsymbol{O})=\psi\left(f_{A}(\boldsymbol{O}) \geq \psi\left(f_{A}(x)\right)=f_{\psi}(x)\right.
$$

for every $x \in X$. On the other hand, for all $x, y, z \in X$ we obtain the following inequalities:

$$
\begin{gathered}
\min \left\{f_{\psi}(x y * z), f_{\psi}(y), r\left(f_{\psi}(x y * z), f_{\psi}(y)\right)\right\}= \\
=\min \left\{\psi\left(f_{A}(x y * z)\right), \psi\left(f_{A}(y)\right), r\left(f_{\psi}\left(f_{A}(x y * z)\right), \psi\left(f_{A}(y)\right)\right)\right\} \leq \\
\leq \min \left\{\psi\left(f_{A}(x y * z), \psi\left(f_{A}(y)\right)\right), r\left(\min \left\{\psi\left(f_{A}(x y * z)\right), \psi\left(f_{A}(y)\right)\right\},\right.\right. \\
\left.\min \left\{\psi\left(f_{A}(x y * z), \psi\left(f_{A}(y)\right)\right\}\right)\right\} \leq \\
\leq \min \left\{\psi\left(f_{A}(x y * z)\right), \psi\left(f_{A}(y)\right), \min \left\{\psi\left(f_{A}(x y * z), \psi\left(f_{A}(y)\right)\right\}\right\}=\right.
\end{gathered}
$$




$$
\begin{gathered}
=\psi\left(\min \left\{f_{A}(x y * z), f_{A}(y)\right\}\right) \leq \psi\left(\operatorname { m i n } \left\{f_{A}(x y * z), f_{A}(y)\right.\right. \\
\left.\left.d\left(\min \left\{f_{A}(x y * z), f_{A}(y)\right\}, \min \left\{f_{A}(x y * z), f_{A}(y)\right\}\right)\right\}\right) \leq \\
\leq \psi\left(\min \left\{f_{A}(x y * z), f_{A}(y), d\left(f_{A}(x y * z), f_{A}(y)\right)\right\}\right) \leq \psi\left(f_{A}(x z)\right)=f_{\psi}(x z),
\end{gathered}
$$

which means that $B$ is a transversal lower BCC-ideal of $X$. If $\psi\left(f_{A}(\boldsymbol{O})\right)=b$, then clearly the set $B$ is normal. The proof is complete.

In analogous with the preceding facts, a transversal upper set $A$ in $X$ is called transversal upper BCC-ideal of $X$ if $f_{A}(\boldsymbol{O}) \leq f_{A}(x)$ and if there is a function $g:[a, b]^{2} \rightarrow[a, b]$ such that

$$
f_{A}(x z) \leq \max \left\{f_{A}(x y * z), f_{A}(y), g\left(f_{A}(x y * z), f_{A}(y)\right)\right\}
$$

for all $x, y, z \in X$. A transversal upper BCC-ideal $A$ of $X$ is said to be normal if there exists $x \in X$ such that $f_{A}(x)=a$.

We notice that the forms of Theorems 9 and 10 are totally analogous hold and for the cases of the transversal upper BCC-ideals.

Also, we notice that, Theorems 9 and 10 are extension of the former results of D u dek and J un [2]. See and Tasković [12].

\section{TRANSVERSAL CONVEX SETS}

In this part we shall introduce the concept of convexity transversal upper or lower sets. This notion appears in much applications involving optimization, pattern classification, economics, and nonlinear programming.

In further, $\mathbb{R}$ denotes the real line, $I$ denotes the interval $[0,1]$ and $D$ is a convex subset of $\mathbb{R}^{n}$.

In further, for the transversal upper or lower sets we consider a special case of the transversal membership funcion (1) or (4) if the lattice $L:=[a, b] \subset \mathbb{R}$ $(a<b ; a, b \in \mathbb{R})$.

The function $g:[a, b]^{2} \rightarrow[a, b]$ is increasing if $a_{i}, b_{i} \in[a, b]$ and $a_{i} \leq b_{i}$ $(i=1,2)$ implies $g\left(a_{1}, a_{2}\right) \leq g\left(b_{1}, b_{2}\right)$. On the other hand, the function $g$ : $[a, b]^{2} \rightarrow[a, b]$ is level increasing if it is increasing and with the property

$$
g(\max \{x, g(x, x)\}, \max \{x, g(x, x)\}) \leq \max \{x, g(x, x)\}
$$

for every $x \in[a, b]$.

A transversal (upper or lower) set $A$ in $\mathbb{R}^{n}$ is convex if for some level increasing function $g:[a, b]^{2} \rightarrow[a, b]$ and $f_{A}: D \rightarrow[a, b]$ the sets

$$
g\left(D_{r}\right):=\left\{x \in D \mid \max \left\{f_{A}(x), g\left(f_{A}(x), f_{A}(x)\right)\right\} \leq \max \{r, g(r, r)\}\right\}
$$

are convex for each number $r \in[a, b]$. 
On the other hand, as an alternative and more concrete definition of convexity is the following form. A transversal (upper or lower) set $A$ in $\mathbb{R}^{n}$ is convex if there is a function $g: f_{A}(D)^{2} \rightarrow[a, b]$ such that

$$
f_{A}(\lambda x+(1-\lambda) y) \leq \max \left\{f_{A}(x), f_{A}(y), g\left(f_{A}(x), f_{A}(y)\right)\right\}
$$

for all $x, y \in D$ and for every $\lambda \in I$, where $f_{A}: D \rightarrow[a, b]$.

We notice that this definition implies that the function $f_{A}: D \rightarrow[a, b]$ must be a transversal (former name general) convex function. These functions are introduced in our former paper (Task ović: Math. Japonica, 37 (1992), 367-372).

To show the equivalence between the above definitions suppose that $A$ is convex in the sense of the second definition (16) and let $x, y \in g\left(D_{r}\right)$. Therefore $x, y \in D$ and

$$
\max \left\{f_{A}(x), g\left(f_{A}(x), f_{A}(x)\right)\right\}, \max \left\{f_{A}(y), g\left(f_{A}(y), f_{A}(y)\right)\right\} \leq \max \{r, g(r, r)\} .
$$

Let $z=\lambda x+(1-\lambda) y$ for $\lambda \in I$. By convexity of $D$ we obtain $z \in D$. Furthemore, by (16) and (17) we have

$$
\begin{aligned}
f_{A}(z) & \leq \max \left\{f_{A}(x), f_{A}(y), g\left(f_{A}(x), f_{A}(y)\right)\right\} \leq \\
& \leq \max \left\{f_{A}(x), f_{A}(y), \max \left\{g\left(f_{A}(x), f_{A}(x)\right), g\left(f_{A}(y), f_{A}(y)\right)\right\} \leq \cdots\right. \\
& \leq \max \{r, g(r, r)\},
\end{aligned}
$$

and thus $f_{A}(z) \leq \max \{r, g(r, r)\}$; and from level increasing of $g:[a, b]^{2} \rightarrow[a, b]$ we obtain the following inequalities

$$
g\left(f_{A}(z), f_{A}(z)\right) \leq g(\max \{r, g(r, r)\}, \max \{r, g(r, r)\}) \leq \max \{r, g(r, r)\} .
$$

This means that is $\max \left\{f_{A}(z), g\left(f_{A}(z), f_{A}(z)\right)\right\} \leq \max \{r, g(r, r)\}$, i.e., $z \in g\left(D_{r}\right)$. Hence $g\left(D_{r}\right)$ is a convex set.

Conversely, suppose that A is convex in the sense of the first definition, i.e., suppose that $g\left(D_{r}\right)$ is a convex set for each number $r \in[a, b]$. Let $z=\lambda x+(1-\lambda) y$ for $\lambda \in I$. Notice that $x, y \in g\left(D_{r}\right)$ for $\max \{r, g(r, r)\}=\max \left\{f_{A}(x), f_{A}(y), g\left(f_{A}(x), f_{A}(y)\right)\right\}$, and since, by assumption, $g\left(D_{r}\right)$ is convex, so that $z \in g\left(D_{r}\right)$. Therefore, we obtain the following facts

$$
\begin{aligned}
f_{A}(z) & \leq \max \left\{f_{A}(z), g\left(f_{A}(z), f_{A}(z)\right)\right\} \leq \\
& \leq \max \{r, g(r, r)\}=\max \left\{f_{A}(x), f_{A}(y), g\left(f_{A}(x), f_{A}(y)\right)\right\},
\end{aligned}
$$

i.e., $A$ is convex in the sense of the second definition from (16). The proof of this statement is now complete.

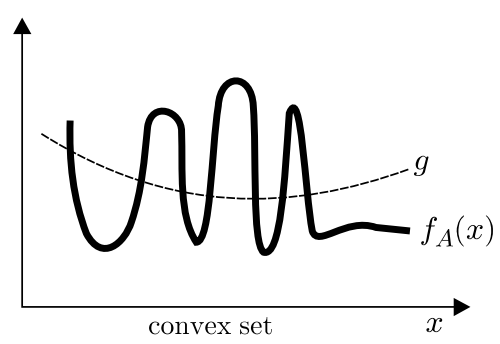

Figure 13

In connection with the preceding, a transversal (upper or lower) set $A$ in $\mathbb{R}^{n}$ is concave if there is a function $d: f_{A}(D)^{2} \rightarrow[a, b]$ such that

$$
f_{A}(\lambda x+(1-\lambda) y) \geq \min \left\{f_{A}(x), f_{A}(y), d\left(f_{A}(x), f_{A}(y)\right)\right\}
$$

for all $x, y \in D$ and for every $\lambda \in I$, where $f_{A}: D \rightarrow[a, b]$. 
A fundamental property of convex transversal upper sets is expressed by the following basic fact.

Theorem 11. Let $A$ and $B$ are transversal upper sets in $\mathbb{R}^{n}$. If $A$ and $B$ are convex, so is their union.

Proof. For $D=A \cup B$, from definition of the union, directly for $z=\lambda x+(1-\lambda) y$ and $\lambda \in I$, we obtain the following equality and inequality

$$
\begin{aligned}
\max \left\{f_{D}(z), g\left(f_{D}(z)\right)\right\} & =\max \left\{f_{A}(z), f_{B}(z), g\left(f_{A}(z)\right), g\left(f_{B}(z)\right)\right\} \leq \\
& \leq \max \left\{f_{A}(z), f_{B}(z), b, b\right\},
\end{aligned}
$$

and thus, since $A$ and $B$ are convex, there are $r: f_{A}(D)^{2} \rightarrow[a, b]$ and $t: f_{B}(D)^{2} \rightarrow[a, b]$ such that we have

$$
\begin{aligned}
f_{A}(z) & \leq \max \left\{f_{A}(x), f_{A}(y), r\left(f_{A}(x), f_{A}(y)\right)\right\} \leq \\
& \leq \max \left\{f_{A}(x), f_{A}(y), b\right\}, f_{B}(z) \leq \max \left\{f_{B}(x), f_{B}(y), t\left(f_{B}(x), f_{B}(y)\right)\right\} \leq \\
& \leq \max \left\{f_{B}(x), f_{B}(y), b\right\} ;
\end{aligned}
$$

i.e., we have the following inequalities

$$
\begin{aligned}
f_{D}(z) & \leq \max \left\{f_{D}(z), g\left(f_{D}(z)\right)\right\} \leq \cdots \leq \\
& \leq \max \left\{\max \left\{f_{A}(x), f_{B}(x), R\left(f_{A}(x), f_{B}(x)\right)\right\}, \max \left\{f_{A}(y), f_{B}(y), R\left(f_{A}(y), f_{B}(y)\right)\right\}\right\},
\end{aligned}
$$

where $R\left(f_{A}(x), f_{B}(x)\right):=\max \left\{f_{A}(x), f_{B}(x), b\right\}$. Thus, from definition of the union for transversal upper sets, we obtain the following inequalities

$$
\begin{aligned}
f_{D}(z) & =f_{A \cup B}(z)=f_{A \cup B}(\lambda x+(1-\lambda) y) \leq \\
& \leq \max \left\{\max \left\{f_{D}(x), q\left(f_{D}(x)\right)\right\}, \max \left\{f_{D}(y), q\left(f_{D}(y)\right)\right\}\right\} \leq \\
& \leq \max \left\{f_{D}(x), f_{D}(y), Q\left(f_{D}(x), f_{D}(y)\right)\right\},
\end{aligned}
$$

where $Q\left(f_{D}(x), f_{D}(y)\right):=\max \left\{q\left(f_{D}(x)\right), q\left(f_{D}(y)\right)\right\}$ and $q\left(f_{D}(x)\right):=R\left(f_{A}(x), f_{B}(x)\right)$. This means that the union $D=A \cup B$ is a convex set. The proof is complete.

As an immediate analogy, of the preceding statement, for transversal lower sets directly we have the following fact.

Theorem 12. Let $A$ and $B$ are transversal lower sets in $\mathbb{R}^{n}$. If $A$ and $B$ are concave, so is their intersection.

The proof of this statement is very similar and totally analogous to the preceding proof of Theorem 11, and thus we omit it.

Transversal numbers. In connection with the preceding facts of $u\left(f_{A}, g\left(f_{A}\right)\right), l\left(f_{A}, d\left(f_{A}\right)\right)$ and the convexity we give the following definition of transversal numbers.

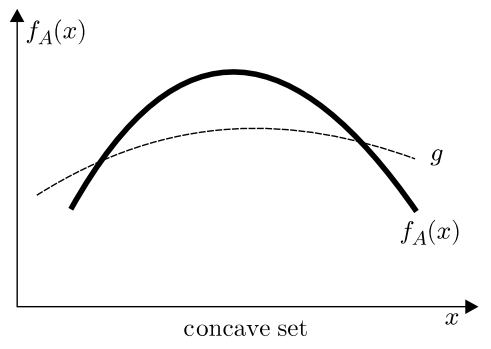

Figure 14 
In this sense, let $L:=[a, b]$, for $a \prec b$, be a lattice. The set $A \subset X$ is said to be tranversal upper normalized if there is a unique element $\xi \in X$ such that

$$
\max \left\{f_{A}(\xi), g\left(f_{A}(\xi)\right)\right\}=b
$$

We notice that this fact implies that is $u[\operatorname{thg} t(A)]=b$. On the other hand, a transversal upper number is a convex transversal upper normalized set $A$ of the real line $\mathbb{R}$, i.e., if (18) and if there is a function $g: f_{A}(\mathbb{R})^{2} \rightarrow[a, b] \subset \mathbb{R}$ such that

$$
f_{A}(y) \leq \max \left\{f_{A}(x), f_{A}(z), g\left(f_{A}(x), f_{A}(z)\right)\right\}
$$

where $x<y<z$ for all $x, y, z \in \mathbb{R}$ and $f_{A}: \mathbb{R} \rightarrow[a, b] \subset \mathbb{R}$.

Also, the set $A \subset X$ is said to be transversal lower normalized if there is a unique element $\eta \in X$ such that

$$
\min \left\{f_{A}(\eta), d\left(f_{A}(\eta)\right)\right\}=a
$$

This fact implies that is $l[\operatorname{thg} t(A)]=a$. In connection with this, a transversal lower number is a concave transversal lower normalized set $A$ of the real line $\mathbb{R}$, i.e., if (19) and if there is a function $d: f_{A}(\mathbb{R})^{2} \rightarrow[a, b] \subset \mathbb{R}$ such that

$$
f_{A}(y) \geq \min \left\{f_{A}(x), f_{A}(z), d\left(f_{A}(x), f_{A}(z)\right)\right\}
$$

where $x<y<z$ for all $x, y, z \in \mathbb{R}$ and $f_{A}: \mathbb{R} \rightarrow[a, b] \subset \mathbb{R}$.

In further, a variant calculation with transversal (upper and lower) numbers we give in the next section of this paper as an abstract calculation with algebras.

In this sense, as for adding of incomplete spaces, we have a completeness rule for transversal sets. Let $f$ be a mapping of $X$ into $Y$, where $X$ and $Y$ are transversal sets. Then for $A \subset X$ we have

$$
[f(A)](y):=\sup \left\{\psi\left[f_{A}(x), g\left(f_{A}(x)\right)\right]: x \in X, y=f(x)\right\},
$$

or

$$
[f(A)](y):=\inf \left\{\psi\left[f_{A}(x), d\left(f_{A}(x)\right)\right]: x \in X, y=f(x)\right\}
$$

where $\psi \in\{\sup , \inf \}$. For further facts of the transversal numbers and the transversal completeness rule see Tasković [12].

\section{TRANSVERSAL INTEGRALS}

In this section we consider a concept of a transversal integral as an extension of the former concept of a classical integral (using minimax results of parth 2).

The classical concept of integration of a real-valued function over a closed interval can be generalizad in the following sense.

Let $X$ be a nonempty set and let $f$ be a function from $X$ into $L:=(L, \preccurlyeq)$. The transversal upper integral of the function $f$ with respect to a function $g: L \rightarrow L$, in notation $\int_{A \subset X} f \& g$, is defined by

$$
\int_{A \subset X} f \& g=\min _{\substack{x \in A \subset X \\\left(\text { or } f_{A}, g\left(f_{A}\right) \in L\right)}} \sup \left\{f_{A}(x), g\left(f_{A}(x)\right)\right\}
$$

where $f_{A}: X \rightarrow L$ for $A \subset X$. 
On the other hand, the transversal lower integral of the function $f$ with respect to a function $d: L \rightarrow L$, in notation $\int_{A \subset X} f \& d$, is defined by

$$
\int_{A \subset X} f \& d=\max _{\substack{x \in A \subset X \\\left(\text { or } f_{A}, d\left(f_{A}\right) \in L\right)}} \inf \left\{f_{A}(x), d\left(f_{A}(x)\right)\right\},
$$

where $f_{A}: X \rightarrow L$ for $A \subset X$. If

$$
\int_{A \subset X} f \& g=\int_{A \subset X} f \& d=\int_{A \subset X} f \&(g, d):=M_{A}(f),
$$

then we call that $M_{A}(f)$ is a transversal integral of the function $f: X \rightarrow L$ with respect to a function $(g, d): L \rightarrow L$.

We notice that if $g: L \rightarrow L$ is a decreasing mapping, then (from Theorem 1) for $d=g$ there is a transversal integral $M_{A}(f)$.

For first example, we now consider briefly the classical Riemann integral via transversal integrals.

Let $[a, b] \subset \mathbb{R}(a<b)$ and $f:[a, b] \rightarrow \mathbb{R}$ be a continuous function. For a partition $\left(P_{n}\right): a=x_{0}<$ $x_{1}<\cdots<x_{n}=b$, let us define $m_{k}=\inf f\left(x_{k}\right)$ and $M_{k}=\sup f\left(x_{k}\right)$ for $k=1, \ldots, n$ on $\left[x_{k-1}, x_{k}\right]$. Then, defining the upper sum

$$
S\left(P_{n}\right)=\sum_{k=1}^{n} M_{k}\left(x_{k}-x_{k-1}\right)
$$

and the lower sum

$$
\sigma\left(P_{n}\right)=\sum_{k=1}^{n} m_{k}\left(x_{k}-x_{k-1}\right)
$$

we obtain a transversal upper integral of the function $f$ in form for the function of the set $A \subset[a, b]$ for $f_{A}\left(x_{k}\right):=\sum_{k=1}^{n} f\left(x_{k}\right)\left(x_{k}-x_{k-1}\right)$ with the respect to a function $g\left(f_{A}\left(x_{k}\right)\right):=S\left(P_{n}\right)$ in the following form as

$$
\begin{aligned}
& \int_{\begin{array}{c}
{[a, b]} \\
(\text { or on } \mathbb{R})
\end{array}} f \& g=\min _{f_{A}, g\left(f_{A}\right) \in \mathbb{R}} \sup \left\{\sum_{k=1}^{n} f\left(x_{k}\right)\left(x_{k}-x_{k-1}\right), S\left(P_{n}\right)\right\}= \\
& =\min _{n \in \mathbb{N}} S\left(P_{n}\right)=\lim _{\left(\text {or } \max \left\{d_{1}, \ldots, d_{n}\right\} \rightarrow 0\right)} S\left(P_{n}\right)=\int_{a}^{b} f(x) d x,
\end{aligned}
$$

where $d_{k}=x_{k}-x_{k-1}$, for $k=1, \ldots, n$. Also, a transversal lower integral of the function $f$ in the form for the function of the set $A \subset[a, b]$ for $f_{A}\left(x_{k}\right):=\sum_{k=1}^{n} f\left(x_{k}\right)\left(x_{k}-x_{k-1}\right)$ with respect to a function $d\left(f_{A}\left(x_{k}\right)\right):=\sigma\left(P_{n}\right)$ is

$$
\begin{aligned}
& \int_{[a, b]} f \& d=\max _{f_{A}, d\left(f_{A}\right) \in \mathbb{R}} \inf \left\{\sum_{k=1}^{n} f\left(x_{k}\right)\left(x_{k}-x_{k-1}\right), \sigma\left(P_{n}\right)\right\}= \\
& \text { (or on } \mathbb{R} \text { ) } \\
& =\max _{n \in \mathbb{N}} \sigma\left(P_{n}\right)=\quad \lim _{n \rightarrow \infty} \quad \sigma\left(P_{n}\right)=\int_{a}^{b} f(x) d x . \\
& \left(\text { or } \max \left\{d_{1}, \ldots, d_{n}\right\} \rightarrow 0\right)
\end{aligned}
$$

We now consider briefly the classical Riemann-Stieltjes integral via transversal integrals.

In this sense, let $r$ be a bounded variation real valued function on the closed and bounded interval $[a, b] \subset \mathbb{R}$, and let $f:[a, b] \rightarrow \mathbb{R}$ be a continuous function. 
For a partition $\left(P_{n}\right): a=x_{0}<x_{1}<\cdots<x_{n}=b$, let us define $M_{k}=\sup r(x)$ and $m_{k}=\inf r(x)$ for $x_{k-1}<x<x_{k}(k=1, \ldots, n)$. Then, defining the upper sum

$$
U\left(P_{n}\right)=\sum_{k=1}^{n} M_{k}\left[f\left(x_{k}\right)-f\left(x_{k-1}\right)\right]
$$

and the lower sum

$$
L\left(P_{n}\right)=\sum_{k=1}^{n} m_{k}\left[f\left(x_{k}\right)-f\left(x_{k-1}\right)\right],
$$

we obtain a transversal upper integral of the function $f$ in the form for the function of the set $A \subset[a, b]$ for $f_{A}\left(x_{k}\right):=\sum_{k=1}^{n} r\left(x_{k}\right)\left[f\left(x_{k}\right)-f\left(x_{k-1}\right)\right]$ with respect to a function $g\left(f_{A}\left(x_{k}\right)\right):=U\left(P_{n}\right)$ in the following form as

$$
\begin{aligned}
& \int_{[a, b]} f \& g=\min _{f_{A}, g\left(f_{A}\right) \in \mathbb{R}} \sup \left\{\sum_{k=1}^{n} r\left(x_{k}\right)\left[f\left(x_{k}\right)-f\left(x_{k-1}\right)\right], U\left(P_{n}\right)\right\}= \\
& \begin{aligned}
\text { or on } \mathbb{R}) \\
=\min _{n \in \mathbb{N}} U\left(P_{n}\right)=\lim _{n \rightarrow \infty} U\left(P_{n}\right)=\int_{a}^{b} f(x) d r(x),
\end{aligned}
\end{aligned}
$$

i.e., we obtain Riemann-Stieltjes integral.

In analogous with this, a transversal lower integral of the function $f$ in the form for the function of the set $A \subset[a, b]$ for $f_{A}\left(x_{k}\right):=\sum_{k=1}^{n} r\left(x_{k}\right)\left[f\left(x_{k}\right)-f\left(x_{k-1}\right)\right]$ with respect to a function $d\left(f_{A}\left(x_{k}\right)\right):=L\left(P_{n}\right)$ is

$$
\begin{aligned}
& \int_{[a, b]} f \& d=\max _{f_{A}, d\left(f_{A}\right) \in \mathbb{R}} \inf \left\{\sum_{k=1}^{n} r\left(x_{k}\right)\left[f\left(x_{k}\right)-f\left(x_{k-1}\right)\right], L\left(P_{n}\right)\right\}= \\
& (\text { or on } \mathbb{R}) \\
& =\max _{n \in \mathbb{N}} L\left(P_{n}\right)=\lim _{n \rightarrow \infty} L\left(P_{n}\right)=\int_{a}^{b} f(x) d r(x) .
\end{aligned}
$$

For further example, we now consider briefly the fuzzy Sugeno integral via transversal lower fuzzy integral.

That is to say, let $f$ be a function from $X$ to $[0,1]$. Then for the functions $f_{A}:=f$ and $d\left(f_{A}\right):=$ $\min \left[\alpha, r\left(A \cap H_{\alpha}\right)\right]$, where $H_{\alpha}=\{x: f(x) \geq \alpha\}$ and $r \in[0,1]$ a fuzzy measure from Suge no [5] we have the following transversal lower (fuzzy) integral as

$$
\begin{aligned}
\int_{A \subset X} f \& d & =\max _{\substack{f_{A}, d\left(f_{A}\right) \in[0,1] \\
(\text { or } x \in A \subset X)}} \inf \left\{f_{A}(x), \min \left[\alpha, r\left(A \cap H_{\alpha}\right)\right]\right\}= \\
& =\max _{\alpha \in[0,1]} \min \left[\alpha, r\left(A \cap H_{\alpha}\right)\right]:=\text { Sugeno's fuzzy integral. }
\end{aligned}
$$

The analogy of this we have and an upper variant of the preceding form integral. Namely, for the functions $f: X \rightarrow[0,1], f_{A}:=f$ and $g\left(f_{A}\right):=\max \left[\alpha, r\left(A \cap H^{\alpha}\right)\right]$, where $H^{\alpha}=\{x: f(x) \leq \alpha\}, f_{A}:=f$ and $r \in[0,1]$ a fuzzy measure from D u b o is-P r a d e [1], we obtain the following transversal upper (fuzzy) integral as

$$
\begin{aligned}
\int_{A \subset X} f \& g= & \min _{\substack{f_{A}, g\left(f_{A}\right) \in[0,1] \\
(\text { or } x \in A \subset X)}} \sup \left\{f_{A}(x), \max \left[\alpha, r\left(A \cap H^{\alpha}\right)\right]\right\}= \\
= & \min _{\alpha \in[0,1]} \max \left[\alpha, r\left(A \cap H^{\alpha}\right)\right],
\end{aligned}
$$

which is similar with the integral of Kandel, i.e., with an integral from Kandel [5].

On the other hand, the analogy of the preceding facts with a Lebesgue integral can be clearly exhibited as follows. Let $E_{1}, \ldots, E_{n}$ be an ordinary partition of $X$ and assume

$$
f(x)=\sum_{k=1}^{n} a_{k} \mu_{E_{k}}(x),
$$


where $x \mapsto \mu_{E k}(x)$ is the crisp characterustic function of $E_{k}(k=1, \ldots, n)$. Let $m$ be a Lebesgue measure on $(X, \mathcal{P}(X))$. The Lebesgue integral of $f$ over $A$ is the following form

$$
\int_{A} f d m=\sum_{k=1}^{n} a_{k} m\left(A \cap E_{k}\right):=\max _{k=1, \ldots, n} \min \left\{a_{k}, m\left(A \cap E_{k}\right)\right\} .
$$

Let $F_{k}=\cup_{j=k}^{n} E_{j}$ and defining $f_{A}:=f$ and $d\left(f_{A}(x)\right):=\max _{k=1, \ldots, n} \min \left[a_{k}, \mu_{F_{k}}(x)\right]$ we obtain the following form of a transversal lower integral as

$$
\begin{aligned}
\int_{A} f \& d & =\max _{x \in A} \inf \left\{f_{A}(x), \max _{k=1, \ldots, n} \min \left[a_{k}, \mu_{F_{k}}(x)\right]\right\}= \\
& =\max _{k=1, \ldots, n} \min \left[a_{k}, m\left(A \cap F_{k}\right)\right] .
\end{aligned}
$$

On the other hand, a vector lattice $\mathcal{D}$ is a vector space of real-valued functions on the nonempty set $X$ such that $f$ in $\mathcal{D}$ implies $|f|$ is in $\mathcal{D}$ and $\inf \{f, 1\}$ is in $\mathcal{D}$ for all $f \in \mathcal{D}$.

In this sense, a Daniell integral $I(f)$ on $\mathcal{D}$ is a positive linear functional on $\mathcal{D}$ such that if $\left(f_{n}\right)$ is a nonincreasing sequence in $\mathcal{D}$ converging to zero, then $I\left(f_{n}\right)$ converges to zero.

We notice that if $I(f)$ is a positive linear functional on $\mathcal{D}$, then the preceding condition holds if and only if:if $\left(f_{n}\right)$ is a nondecreasing sequence in $\mathcal{D}$ with $f=\sup _{n \in \mathbb{N}} f_{n}$ in $\mathcal{D}$, then $I(f)=\sup _{n \in \mathbb{N}} I\left(f_{n}\right)$.

We now consider briefly the Daniell integral via transversal integral. Then, from the precding facts, we obtain a transversal lower integral of the function $f \in \mathcal{D}$ with the respect to a function $d\left(f_{A}\right):=I\left(f_{n}\right)$, where $f_{A}:=I(f)$, in the following form as

$$
\int_{A} f \& d=\max _{I(f), I\left(f_{n}\right) \in \mathbb{R}} \inf \left\{I(f), I\left(f_{n}\right)\right\}=\max _{n \in \mathbb{N}} I\left(f_{n}\right)=\lim _{n \rightarrow \infty} I\left(f_{n}\right),
$$

i.e., a lower transversal integral of the function $f \in \mathcal{D}$ is equal of the Daniell inteegral of the function $f \in \mathcal{D}$.

In connection with the preceding facts, we notice that in 1953 in the theory of capacities G. C h o qu et is consider the following integral of the form

$$
\text { (Choquet) } \int h d m=\int_{0}^{\infty} m(\{x: h(x) \geq r\}) d r
$$

which, also, can be consider as a transversal integral in the preceding sense. For further facts of this see: Tasković [12].

In connection with the preceding facts, the preceding concept of transversal integration of a function can be generalized in the following sense.

Let $X_{1}, \ldots, X_{n}$ are nonempty sets and let $f_{1}, \ldots, f_{n}$ are functions from $X_{1}, \ldots, X_{n}$ into $L:=(L, \preccurlyeq)$, respectively.

The $n$-transversal upper integral of the functions $f_{k}: X_{k} \rightarrow L$ for $k=$ $1, \ldots, n$ with respect to a function $g: L^{n} \rightarrow L(n \in \mathbb{N}$ is fixed) is defined by

$$
\begin{gathered}
\int_{X_{1}} \int_{X_{2}} \ldots \int_{X_{n}}\left(f_{1}, \ldots, f_{n}\right) \& g= \\
=\min _{\substack{x_{1} \in X_{1}, \ldots, x_{n} \in X_{n} \\
\left(\text { or } \bar{f}_{1}, \ldots, \bar{f}_{n}, g \in L\right)}} \sup \left\{\bar{f}_{1}\left(x_{1}\right), \ldots, \bar{f}_{n}\left(x_{n}\right), g\left(\bar{f}_{1}\left(x_{1}\right), \ldots, \bar{f}_{n}\left(x_{n}\right)\right)\right\},
\end{gathered}
$$

where $\bar{f}_{i}: A_{i} \rightarrow L$ for $A_{i} \subset X_{i}(i=1, \ldots, n)$. 
On the other hand, the $\boldsymbol{n}$-transversal lower integral of the functions $f_{k}$ : $X_{k} \rightarrow L$ for $k=1, \ldots, n$ with respect to a function $d: L^{n} \rightarrow L$ is defined by

$$
=\max _{\substack{x_{1} \in X_{1}, \ldots, x_{n} \in X_{n} \\\left(\operatorname{or} \bar{f}_{1}, \ldots, \bar{f}_{n}, d \in L\right)}} \inf \left\{\bar{f}_{1}\left(x_{1}\right), \ldots, \bar{f}_{n}\left(x_{n}\right), d\left(\bar{f}_{1}\left(x_{1}\right), \ldots, \bar{f}_{n}\left(x_{n}\right)\right)\right\},
$$

where $\bar{f}_{i}: A_{i} \rightarrow L$ for $A_{i} \subset X_{i}(i=1, \ldots, n)$. In connection with the preceding definitions, if

$$
\begin{gathered}
\int_{X_{1}} \int_{X_{2}} \cdots \int_{X_{n}}\left(f_{1}, \ldots, f_{n}\right) \& d=\int_{X_{1}} \int_{X_{2}} \cdots \int_{X_{n}}\left(f_{1}, \ldots, f_{n}\right) \& g= \\
=\int_{X_{1}} \int_{X_{2}} \cdots \int_{X_{n}}\left(f_{1}, \ldots, f_{n}\right) \&(g, d):=M_{n}(f),
\end{gathered}
$$

then we call that $M_{n}(f)$ is a $\boldsymbol{n}$-transversal integral of the functions $f_{k}: X_{k} \rightarrow L$ for $k=1, \ldots, n$ with respect to a function $(g, d): L^{n} \rightarrow L$.

We notice that if $g: L^{n} \rightarrow L$ is a decreasing mapping, then (from (Uk)) there is a $n$-transversal integral $M_{n}(f)$ if and only if

$$
\overline{f_{1}}\left(a_{1}\right)=\cdots=\overline{f_{n}}\left(a_{n}\right):=\xi=g(\xi, \ldots, \xi)
$$

for some $a_{1} \in X_{1}, \ldots, a_{n} \in X_{n}$.

On the other hand, in connection with the preceding facts and from the Statement of Separation (Theorem 3), we have the following characterization of the transversal integrals.

Proposition 1. Let $X$ be a nonempty set and let $f$ be a function from $X$ into $L:=(L, \preccurlyeq)$ which is with the property of local comparability. Then there is a transversal integral of the function $f$ with respect to a function $g: L \rightarrow L$ denoted by

$$
M_{A}(f)=\int_{A \subset X} f \& g
$$

if and only if there exists a decreasing function $\psi: L^{2} \rightarrow L$ such that

$$
\begin{aligned}
p(x):= & \inf \left\{f_{A}(x), g\left(f_{A}(x)\right)\right\} \leq \psi(p(x), q(y)) \leq \\
& \leq q(y):=\sup \left\{f_{A}(x), g\left(f_{A}(x)\right)\right\},
\end{aligned}
$$

where $f_{A}: X \rightarrow L$ for $A \subset X$, for all $x, y \in X$ and if there is $\xi \in L$ such that $\xi \cap p(X)$ and $\xi \cap q(Y)$ are nonempty sets.

There proof of this statement is a directly consequence of Theorem 3 from the former part of Minimax facts on transversal sets. 


\section{TRANSVERSAL INTEGRABILITY}

In this part we consider and to compare the facts on Lebesque and transversal integrals. In this sense, the following statements are essential.

Proposition 2. Let $X$ be a nonempty set, $P:=(P, \preccurlyeq)$ be a partially ordered set, let $A \subset X$, and let $f_{n}: A \rightarrow P$ (for $n \in \mathbb{N}$ ) be a sequence of functions such that

$$
f_{1}(x) \preccurlyeq f_{2}(x) \preccurlyeq \cdots \preccurlyeq f_{n}(x) \preccurlyeq \cdots \preccurlyeq f(x),
$$

where $f: A \rightarrow P$. If $\mathcal{D}: P \rightarrow P$ is an increasing mapping, then there exists lower transversal integral of the function $f$ and

$$
\int_{A \subset X} f \& d=\max _{n \in \mathbb{N}} \mathcal{D}\left(f_{n}\right)=\lim _{n \rightarrow \infty} \mathcal{D}\left(f_{n}\right) ;
$$

and, on the other hand, if $\mathcal{D}: P \rightarrow P$ is a decreasing mapping, there exists upper transversal integral of the function $f$ and

$$
\int_{A \subset X} f \& g=\min _{n \in \mathbb{N}} \mathcal{D}\left(f_{n}\right)=\lim _{n \rightarrow \infty} \mathcal{D}\left(f_{n}\right) .
$$

A brief proof of this statement in transversal integrability may be found (on a standard manner) in Tasković [12]. On the other hand, we notice that Proposition 2 is an extension of the classical B e p po Levi (1875-1961) statement in Lebesgue's theory.

Proposition 3. Let $X$ be a nonempty set, $P:=(P, \preccurlyeq)$ be a partially ordered set with the property that every increasing sequence in $P$ has a supremum and let $A \subset X$. If $f_{n}: A \rightarrow P$ (for $n \in \mathbb{N}$ ) is an increasing sequence of functions and $\mathcal{D}: P \rightarrow P$ is an increasing mapping, then there exists a lower transversal integrable function $f: A \rightarrow P$.

A brief proof of this statement may be found in: Tasković [12].

Corollary 4. Let $X$ be a nonempty set, $L:=(L, \preccurlyeq)$ be a lattice, let $A \subset X$ and let $f_{n}: A \rightarrow L$ (for $n \in \mathbb{N}$ ) be a sequence of functions such that $f_{n} \preccurlyeq f_{0}$ for every $n \in \mathbb{N}$, where $f_{0}: A \rightarrow L$. If $\mathcal{D}: L \rightarrow L$ is an increasing mapping, then there exists a lower transversal integrable function $f: A \rightarrow L$. On the other hand, if $\mathcal{D}: L \rightarrow L$ is a decreasing mapping, then there exists an upper transversal integrable function $f: A \rightarrow L$.

A brief proof of this fact, applying Proposition 2 to the sequence $y_{k}=\sup \left\{f_{1}, \ldots, f_{k}\right\}$ for $k \in \mathbb{N}$, may be found in: Tasković [12].

Corollary 5. Let $X$ be a nonempty set, $L:=(L, \preccurlyeq)$ be a lattice, let $A \subset X$ and let $f_{n}: A \rightarrow L$ (for $n \in \mathbb{N}$ ) be a sequence of functions such that $f_{0} \preccurlyeq f_{n}$ for every $n \in \mathbb{N}$ where $f_{0}: A \rightarrow L$. If $\mathcal{D}: L \rightarrow L$ is an increasing mapping, then there exists an upper transversal integrable function $f: A \rightarrow L$. On the other hand, if $\mathcal{D}: L \rightarrow L$ is a decreasing mapping, then there exists a lower transversal integrable function $f: A \rightarrow L$. 
A brief proof of this fact, applying Proposition 2 to the sequence $y_{k}=\inf \left\{f_{1}, \ldots, f_{k}\right\}$ for $k \in \mathbb{N}$, may be found in: Tasković [12].

Further facts. Let $X$ be a nonempty set, $P:=(P, \preccurlyeq)$ be a partially ordered set, and let $\mathcal{R} \subset P^{A}$ for $A \subset X$. In this sense, a set $\mathcal{R}$ is called transversal grate if $x, y \in \mathcal{R}$ implies that

$$
\inf \{x, y\} \in \mathcal{R} \quad \text { and } \quad \sup \{x, y\} \in \mathcal{R} .
$$

In connection with this, a set $\mathcal{R}$ is called transversal $\sigma$-grate of the functions $f_{n}: A \rightarrow P($ for $n \in \mathbb{N} \cup\{0\})$ if

$$
f_{n} \preccurlyeq f_{0}(\text { for } n \in \mathbb{N}) \text { implies } \sup \left\{f_{n}: n \in \mathbb{N}\right\} \in \mathcal{R}
$$

and

$$
f_{n} \succcurlyeq f_{0}(\text { for } n \in \mathbb{N}) \text { implies } \quad \inf \left\{f_{n}: n \in \mathbb{N}\right\} \in \mathcal{R},
$$

where $f_{n} \in \mathcal{R}$ (for $n \in \mathbb{N} \cup\{0\}$ ).

In this sense, from the preceding facts, we can write that Corollaries 4 and 5 to realize extension of transversal integrability and on transversal $\sigma$-grate.

Also, a function $f: A \rightarrow P$ is called lower transversal integrable if there exists an increasing sequence of functions $f_{n}: A \rightarrow P$ (for $n \in \mathbb{N}$ ) such that $f=\lim _{n \rightarrow \infty} f_{n}\left(=\sup _{n \in \mathbb{N}} f_{n}\right)$. The set of all lower transversal integrable functions denoted by $\mathcal{T}_{*}$.

If $f \in \mathcal{T}_{*}$ is a lower transversal integrable function and $f=\sup _{n \in \mathbb{N}} f_{n}$ for $f_{1} \preccurlyeq f_{2} \preccurlyeq \cdots \preccurlyeq f_{n} \preccurlyeq \cdots$, then lower transversal integral of the following form

$$
D(f)=\int_{A} f \& d=\sup _{n \in \mathbb{N}} \mathcal{D}\left(f_{n}\right) ;
$$

and, i.e., if $f \in \mathcal{T}_{*}$ with the property $(\mathrm{Mb})$, then there exists $D(f)$ from Proposition 2 .

The function $f: A \rightarrow P$ is called transversal lower function if there exists an increasing sequence of functions $\left\{f_{n}\right\}_{n \in \mathbb{N}}$ such that

$$
f_{1} \preccurlyeq f_{2} \preccurlyeq \cdots \preccurlyeq f_{n} \preccurlyeq \cdots \preccurlyeq f \quad(\text { for } n \in \mathbb{N})
$$

and $\sup _{n \in \mathbb{N}} f_{n}\left(=\lim _{n \rightarrow \infty} f_{n}\right)=f$. The set of all transversal lower functions denoted by $\mathcal{T}$.

Further, a function $f: A \rightarrow P$ is called upper transversal integrable if there exists a decreasing sequence of functions $f_{n}: A \rightarrow P($ for $n \in \mathbb{N}$ ) such that $f=\lim _{n \rightarrow \infty} f_{n}\left(=\inf _{n \in \mathbb{N}} f_{n}\right)$. The set of all upper transversal integrable functions denoted by $\mathcal{T}^{*}$.

If $f \in \mathcal{T}^{*}$ is an upper transversal integrable function and $f=\inf _{n \in \mathbb{N}} f_{n}$ for $\cdots \preccurlyeq f_{n} \preccurlyeq \cdots \preccurlyeq f_{2} \preccurlyeq f_{1}$, then upper transversal integral of the following form

$$
G(f)=\int_{A} f \& g=\inf _{n \in \mathbb{N}} \mathcal{D}\left(f_{n}\right) ;
$$

i.e., in this sense, this means that if $f \in \mathcal{T}^{*}$ with the property

$$
f \preccurlyeq \cdots \preccurlyeq f_{n} \preccurlyeq \cdots \preccurlyeq f_{2} \preccurlyeq f_{1} \quad(\text { for } n \in \mathbb{N}) \text {, }
$$


then there exists $G(f)$ from Proposition 2 .

The function $f: A \rightarrow P$ is called transversal upper function if there exists a decreasing sequence of functions $\left\{f_{n}\right\}_{n \in \mathbb{N}}$ such that (Db) and if holds that is $\inf _{n \in \mathbb{N}} f_{n}\left(=\lim _{n \rightarrow \infty} f_{n}\right)=f$. The set of all transversal upper functions denoted by $\mathcal{G}$.

Proposition 4. Let $X$ be a nonempty set, $L:=(L, \preccurlyeq)$ be a conditionally complete lattice, let $A \subset X$, and let $f_{n}: A \rightarrow L$ (for $n \in \mathbb{N}$ ) be a sequence of functions such that $f_{0} \preccurlyeq f_{n} \preccurlyeq r_{0}$ for some functions $f_{0}, r_{0}: A \rightarrow L$ and for every $n \in \mathbb{N}$. If $\mathcal{D}: L \rightarrow L$ is an increasing mapping, then there exist functions $p, p_{n}: A \rightarrow L($ for $n \in \mathbb{N})$ such that $p=\lim _{n \rightarrow \infty} p_{n}$ and $\mathcal{D}(p) \succcurlyeq \lim _{n \rightarrow \infty} \mathcal{D}\left(p_{n}\right)$, and there exist functions $q, q_{n}: A \rightarrow L$ (for $n \in \mathbb{N}$ ) such that $q=\lim _{n \rightarrow \infty} q_{n}$ and $\mathcal{D}(q) \preccurlyeq \lim _{n \rightarrow \infty} \mathcal{D}\left(q_{n}\right)$.

A brief proof of this statement may be found in: Tasković [12]. We notice that this statement is directly connection with the classical Fatou's lemma in Lebesgue's theory.

Transversal measurable functions. Let $X$ be a nonempty set, let $A \subset X$, and let $P:=(P, \preccurlyeq)$ be a partially ordered set.

In connection with the preceding, a function $f: A \rightarrow P$ is called lower transversal measurable function if there exists a sequence of functions $f_{n}: A \rightarrow P$ (for $n \in \mathbb{N}$ ) such that $f=\sup _{n \in \mathbb{N}} f_{n}\left(=\lim _{n \rightarrow \infty} f_{n}\right)$. The set of all lower transversal measurable functions denoted by $\mathcal{M}_{d}(A, \mathcal{D})$ or only $\mathcal{M}_{d}$.

Proposition 5. Every lower transversal integrable function $f \in \mathcal{T}_{*}$ is a lower transversal measurable function, i.e., $f \in \mathcal{M}_{d}(A, \mathcal{D})$.

Further, a function $f: A \rightarrow P$ is called upper transversal measurable function if there exists a sequence of function $f_{n}: A \rightarrow P$ (for $n \in \mathbb{N}$ ) such that1 $f=\inf _{n \in \mathbb{N}} f_{n}\left(=\lim _{n \rightarrow \infty} f_{n}\right)$. The set of all upper transversal measurable functions denoted by $\mathcal{M}_{g}(A, \mathcal{D})$ or only $\mathcal{M}_{g}$.

Proposition 6. Every upper transversal integrable function $f \in \mathcal{T}^{*}$ is an upper transversal measurable function, i.e., $f \in \mathcal{M}_{g}(A, \mathcal{D})$.

Extended transversal integrals. In this part we consider an expansion of the preceding concept of transversal integrals.

Let $X$ be a nonempty set and let $f$ be a function from $X$ into $L:=(L, \preccurlyeq)$. The wide transversal upper integral of the function $f$ with respect to a function $g: L \rightarrow L$, in notation $p r . \int_{A \subset X} f \& g$, is defined by

$$
p r . \int_{A \subset X} f \& g=\inf _{\substack{x \in A \subset X \\\left(\operatorname{or} f_{A}, g\left(f_{A}\right) \in L\right)}} \sup \left\{f_{A}(x), g\left(f_{A}(x)\right)\right\},
$$

where $f_{A}: X \rightarrow L$ for $A \subset X$. 
On the other hand, the wide transversal lower integral of the function $f$ with respect to a function $d: L \rightarrow L$, in notation $p r . \int_{A \subset X} f \& d$, is defined by

$$
p r . \int_{A \subset X} f \& d=\sup _{\substack{x \in A \subset X \\\left(\text { or } f_{A}, d\left(f_{A}\right) \in L\right)}} \inf \left\{f_{A}(x), d\left(f_{A}(x)\right)\right\},
$$

where $f_{A}: X \rightarrow L$ for $A \subset X$. If

$$
p r . \int_{A \subset X} f \& g=p r . \int_{A \subset X} f \& d=p r . \int_{A \subset X} f \&(g, d)=p r . M_{A}(f),
$$

then we call that $p r . M_{A}(f)$ is a wide transversal integral of the function $f$ : $X \rightarrow L$ with respect to a function $(g, d): L \rightarrow L$.

In connection with the preceding facts, the preceding concept of wide transversal integration of a function can be generalized in the following sense.

Let $X_{1}, \ldots, X_{n}$ are nonempty sets and let $f_{1}, \ldots, f_{n}$ are functions from $X_{1}, \ldots, X_{n}$ into $L$, respectively for fixed $n \in \mathbb{N}$.

The wide $\boldsymbol{n}$-transversal upper integral of the functions $f_{k}: X_{k} \rightarrow L$ for $k=1, \ldots, n$ with respect to a function $g: L^{n} \rightarrow L$ is defined by

$$
\begin{gathered}
\text { pr. } \int_{X_{1}} \int_{X_{2}} \ldots \int_{X_{n}}\left(f_{1}, \ldots, f_{n}\right) \& g= \\
=\inf _{\substack{x_{1} \in X_{1}, \ldots, x_{n} \in X_{n} \\
\left(\text { or } \bar{f}_{1}, \ldots, \bar{f}_{n}, g \in L\right)}} \sup \left\{\bar{f}_{1}\left(x_{1}\right), \ldots, \bar{f}_{n}\left(x_{n}\right), g\left(\bar{f}_{1}\left(x_{1}\right), \ldots, \bar{f}_{n}\left(x_{n}\right)\right)\right\},
\end{gathered}
$$

where $\bar{f}_{i}: A_{i} \rightarrow L$ for $A_{i} \subset X_{i}(i=1, \ldots, n)$.

On the other hand, the wide $\boldsymbol{n}$-transversal lower integral of the functions $f_{k}: X_{k} \rightarrow L$ for $k=1, \ldots, n$ with respect to a function $d: L^{n} \rightarrow L$ is defined by

$$
\begin{aligned}
& p r . \int_{X_{1}} \int_{X_{2}} \cdots \int_{X_{n}}\left(f_{1}, \ldots, f_{n}\right) \& d= \\
& =\sup _{\substack{x_{1} \in X_{1}, \ldots, x_{n} \in X_{n} \\
\left(\text { or } \bar{f}_{1}, \ldots, \bar{f}_{n}, d \in L\right)}} \inf \left\{\bar{f}_{1}\left(x_{1}\right), \ldots, \bar{f}_{n}\left(x_{n}\right), d\left(\bar{f}_{1}\left(x_{1}\right), \ldots, \bar{f}_{n}\left(x_{n}\right)\right)\right\},
\end{aligned}
$$

where $\bar{f}_{i}: A_{i} \rightarrow L$ for $A_{i} \subset X_{i}(i=1, \ldots, n)$. In connection with the preceding definitions, if

$$
\begin{gathered}
p r \cdot \int_{X_{1}} \cdot \int_{X_{2}} \cdots \int_{X_{n}}\left(f_{1}, \ldots, f_{n}\right) \& d=p r \cdot \int_{X_{1}} \int_{X_{2}} \cdots \int_{X_{n}}\left(f_{1}, \ldots, f_{n}\right) \& g= \\
=p r \cdot \int_{X_{1}} \int_{X_{2}} \cdots \int_{X_{n}}\left(f_{1}, \ldots, f_{n}\right) \&(g, d):=M_{p r}(f),
\end{gathered}
$$

then we call that $M_{p r}$. $(f)$ is a wide $\boldsymbol{n}$-transversal integral of the functions $f_{k}: X_{k} \rightarrow L$ for $k=1, \ldots, n$ with respect to a function $(g, d): L^{n} \rightarrow L$. 


\section{FURTHER ON TRANSVERSAL SETS}

We notice that, from the preceding facts in this paper, we give a technology of an arbitrary set (as atom) via transversal upper and lower sets.

In this sense, every set has three part (or three sides, or three projections) as a bell on Figure 15, or as a coin on Figure 16.

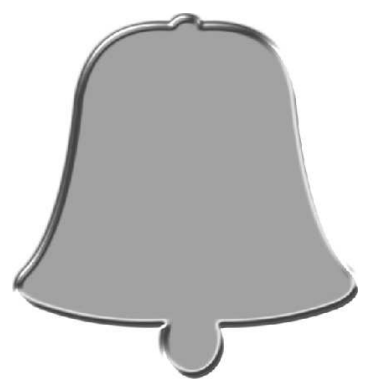

Figure 15

Every set has three microscopic projections as parts which are not see ${ }^{2}$, but this sides existing as three (upper, lower and medial) transversal sets.

Every atom (particle) in physics can be explain as a transversal set which has: positrons (as an upper transversal set), electrons (as a lower transversal set), and neutrons (as a transversal set). This are new facts on atoms in physics as a new realism.

The preceding "three sides" of an arbitrary set to means adequate that there exist three transversal integrals (upper, lower, and medial), see part 9 of this paper. This is an explanation that existing, an example, three Riemann's integrals.

Transversal topology. A transversal topology is a family $\mathcal{J}$ of transversal sets (upper or lower or medial) on the set $X$ satisfying the following facts: $X, \varnothing \in \mathcal{J}$; if $A, B \in \mathcal{J}$, then $A \cap B \in \mathcal{J}$; and if $A_{j} \in \mathcal{J}$ for every $j \in J$ (=index set), then $\cup_{j \in J} A_{j} \in \mathcal{J}$.

In this sense, $(X, \mathcal{J})$ is said to be a transversal topological space (upper or lower or medial).

Every member of $\mathcal{J}$ is called a $\mathcal{J}$-open transversal set (upper or lower or medial). A transversal set (upper or lower or medial) is $\mathcal{J}$-closed iff its complement is $\mathcal{J}$-open.

A transversal set (upper or lower or medial) $\mathcal{O} \in \mathcal{J}$ is a neighborhood of $A$ iff there exists $B \in \mathcal{J}$ such that $A \subset B \subset \mathcal{O}$. The set $A$ (transversal) is open iff for each transversal set $B$ contained in $A, A$ is a neighborhood of $B$.

\footnotetext{
${ }^{2} \mathrm{Ge}$ or g Can tor: I think of a set as a precipice. On the other hand, Le o pold Kronecker brief: Cantor is the corruptor of youth. Rich ard Dedekind: "I think of a set as a closed sack which contains certain specified objects which one doesn't see". David Hilbert in 1925: "No one should ever drive us from the paradise which Cantor created for us". Bertrand Russel: "Thus mathematics may defined as the subject in which we never know that we are talking about, nor whether what we are saying is true".
} 
We notice that the above definition is somewhat different from the ordinary one in that we do not consider here the neighborhood of a point but of a transversal set (upper or lower or medial).

Let $A$ and $B$ be transversal (upper or lower or medial) sets of $\mathcal{J}$ such that $B \subset A$. Then $B$ is said interior to $A$ iff $A$ is a neighborhood of $B$. The interior of $A$, denoted by $\operatorname{Int} t(A)$, is the union of all interior transversal sets $A$. Evident, Int $t(A)$ is the largest open transversal set (upper or lower or medial) contained in $A$; also, $A$ is open if and only if $A=\operatorname{Int} t(A)$.

Let $f$ be a function from $X$ to $Y$, and let $\mathcal{U}$ be a transversal topology on $Y$. The inverse, denoted by $f^{-1}(B)$, of a transversal set $B$ in $Y$ is a transversal set in $X$ whose membership transversal function is

$$
\sup \left\{\psi_{f^{-1}(B)}(x), g\left(\psi_{f^{-1}(B)}(x)\right)\right\} \quad \text { or } \quad \inf \left\{\psi_{f^{-1}(B)}(x), g\left(\psi_{f^{-1}(B)}(x)\right)\right\}
$$

for the function $\psi_{A}: X \rightarrow L:=(L, \preccurlyeq)$, where $L$ is a lattice.

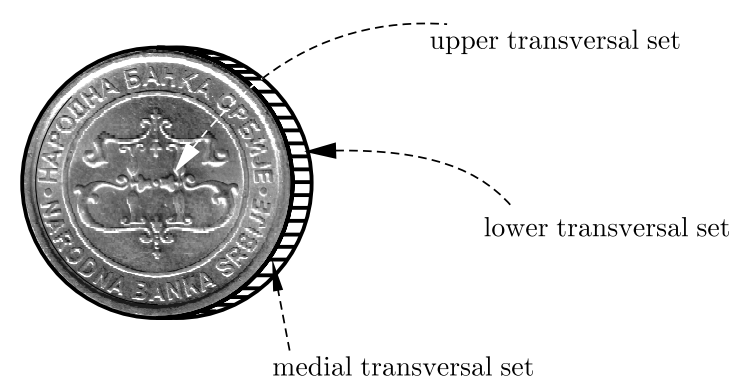

Figure 16

In connection with this, $f$ is said to be transversal continuous (upper or lower or medial) iff the inverse of each $\mathcal{U}$-open set is $\mathcal{J}$-open. Then, for each transversal set $A$ in $X$, the inverse of every neighborhood of $f(A)$ is a neighborhood of $A$.

For further brief facts on transversal compactness (upper, lower, or medial) and transversal connections (upper, lower, or medial) see: Tasković [12].

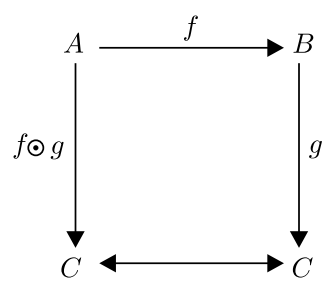

Figure 17

Categories of transversal sets. $A$ category $K$ is a collection of objects, denoted by $\operatorname{obj}(K)$, together with for each pair $(A, B)$ of objects a collection of entities called morphisms. The set of morphisms $f$ between $A$ and $B$ is denoted by $K(A, B)$ and $K(B, C)$ can be composed to make a unique morphism $g \odot f$ in $K(A, C)$. Symbolically, we write and say that "the diagram commutes". 
For $K$ to be a category, the following properties must be satisfied: the composition law of morphisms is associative and for every $A \in \operatorname{obj}(K)$ there is a unique morphism $i d_{A} \in K(A, A)$ such that for every $f: A \rightarrow B$ is $f \odot i d_{A}=i d_{A} \odot f=f$. There $i d_{A}$ is called the identity morphism.

Let Cat $(L)$ denoted category of transversal sets (upper, lower, or medial), where $L$ is a complete lattice. Objects of $\operatorname{Cat}(L)$ are transversal sets (upper, lower, or medial), i.e., pairs $\left(X, f_{A}\right)$, where $X$ is an ordinary set and $f_{A}$ a function from $X$ to $L$ and where $A$ is a transversal set (upper, lower, or medial). Morphisms are ordinary functions $f: X \rightarrow Y$ such that $f_{A}(f) \leq($ or $\geq) r_{A}$, where $\left(X, f_{A}\right)$ and $\left(X, r_{A}\right)$ are objects of set $\operatorname{Cat}(L)$.

Other categories of transversal sets (upper, lower, or medial) include and $\operatorname{Cat}_{f}(L)$ as the category whose objects are pairs $(X, f)$ for $f: X \rightarrow L$ and whose morphisms are transversal relations $\mu_{R}: X \times Y \rightarrow L$ such that

or

$$
\mu_{R}(x, y) \preccurlyeq \sup \{\mu(x), r(y), G(\mu(x), r(y))\}
$$

$$
\mu_{R}(x, y) \succcurlyeq \inf \{\mu(x), r(y), D(\mu(x), r(y))\}
$$

for some give functions $G, D: L^{2} \rightarrow L$, where $(X, \mu),(Y, r)$ in $\operatorname{obj}\left(\operatorname{Cat}_{f}(L)\right)$. Thus $\operatorname{Cat}_{f}(L)$ is the category of transversal sets and transversal relations in the former sense.

A transversal theory is a triple $\mathcal{F}=\{F, r, j\}$; where $F$ is a function from $\operatorname{obj}(K)$ to $\operatorname{obj}(K), r$ is a function from $K(A, F(B)) \times K(B, F(C))$ to $K(A, F(C))$, and $j$ a collection of morphisms $A$ in $F(A)$ for $A$ in $\operatorname{obj}(K)$. It is possible to equip $\mathcal{F}$ with properties such that there is a category $\mathcal{F}(K)$ with $\operatorname{obj}(\mathcal{F}(K))=\operatorname{obj}(K)$ and $\mathcal{F}(K)=K(A, F(B))$ for all $A, B \in o b j(K)$.

A role of this concept is discussed under the name "transversal characters" in a categorical framework. For furher facts of this see: Tasković [12].

An annotation. In further, as a natural step, we considered several fundamental facts on transversal dimension theory on transversal topological spaces. For some facts on transversal dimension see: Tasković [12].

Transversal logic. In connection with the transversal sets we can consider suitable transversal logics (upper, lower, and medial).

In this sense, if $L:=[a, b] \subset \mathbb{R}$ for $a<b$, first, we introduce the negation \rceil $x:=c(x)$ of some statement $x \in L$ as a function defined on $L$ in the following form:

$$
c(x)=b-\min \{x, d(x)\}
$$

or

$$
c(x)=b-\max \{x, g(x)\},
$$

where $d, g: L \rightarrow L$. (Since every set has three sides, thus natural there exist three negation of some statement.)

In this sense, we obtain transversal conjuction, denoted by $x \wedge_{t r} y$, in the following form such that

$$
x \wedge_{t r} y:=\min \{x, y, \psi(x, y)\},
$$


where $\psi: L \times L \rightarrow L$ is a given function.

On the other hand, transversal disjunction, denoted by $x \vee_{t r} y$, is the following form such that

$$
x \vee_{t r} y:=\max \{x, y, \mathcal{D}(x, y)\},
$$

where $\mathcal{D}: L \times L \rightarrow L$ is a given function. (The formules for transversal conjuction and transversal disjunction can be equel for upper and lower negation, for example.)

If $L=[0,1]$, then we obtain $[0,1]$-transversal fuzzy logic; if $L=\{0,1\}$, then we have Boolean logic, i.e., transversal Boolean logic.

Further transversal implication, denoted by $x \Rightarrow_{t r} y$, is the following form such that

$$
x \Rightarrow_{\text {tr }} y:=\max \{b-\min \{x, d(x)\}, y, \psi(b-\min \{x, d(x)\}, y)\},
$$

where $d: L \rightarrow L$ and $\psi: L \times L \rightarrow L$ are given functions.

In connection with the preceding facts of transversal logic we have and the following $n$-rules. In this sense, transversal $\boldsymbol{n}$-conjuction of statements $x_{1}, \ldots, x_{n}$ is the following form such that

$$
\wedge_{t r}\left(x_{1}, \ldots, x_{n}\right):=\min \left\{x_{1}, \ldots, x_{n}, \psi\left(x_{1}, \ldots, x_{n}\right)\right\},
$$

where $\psi: L^{n} \rightarrow L$ (for a fixed $n \in \mathbb{N}$ ) is a given function. Also, transversal $n$-disjunction of statements $x_{1}, \ldots, x_{n}$ is the following form such that

$$
\vee_{t r}\left(x_{1}, \ldots, x_{n}\right):=\max \left\{x_{1}, \ldots, x_{n}, \mathcal{F}\left(x_{1}, \ldots, x_{n}\right)\right\},
$$

where $\mathcal{F}: L^{n} \rightarrow L$ (for a fixed $n \in \mathbb{N}$ ) is a given function. Adequate, as in the preceding cases, for the negation of statements $x_{1}, \ldots, x_{n}$ we have the following form such that

$$
c\left(x_{1}, \ldots, x_{n}\right)=b-\min \left\{x_{1}, \ldots, x_{n}, d\left(x_{1}, \ldots, x_{n}\right)\right\},
$$

or

$$
c\left(x_{1}, \ldots, x_{n}\right)=b-\max \left\{x_{1}, \ldots, x_{n}, g\left(x_{1}, \ldots, x_{n}\right)\right\},
$$

where $d, g: L^{n} \rightarrow L$ (for a fixed $n \in \mathbb{N}$ ) are given functions.

Further, in this sense, we introduce and transversal $n$-implication. For further facts on transversal $n$-logics see: Tasković [12].

\section{REFERENCES}

[1] D. Dubois and H. Prade, Fuzzy Sets and Systems - Theory and Applications, Academic Press, New York, 1980.

[2] W. A. Dudek and Y.B. Jun, Normalizations of fuzzy BCC-ideals in BCC-algebras, Math. Moravica, 3(1999), 17-24.

[3] K. Iséki and S. Tanaka, Ideal theory of BCK-algebras, Math. Japonica, 21(1976), 352-366.

[4] A. Kandel, Fuzzy statistics and forecast evaluation, IEEE Trans. Syst., Man Cybern, 8(1978), 396-401. 
[5] M. Sugeno, Theory of Fuzzy Integral and Its Applications, Ph. D. Thesis, Tokyo Inst. of Technol., Tokyo, 1974.

[6] H. Tanaka and H. Ishibuchi, Identification of possibilistic linear systems by quadratic membership functions, Fuzzy Sets and Systems, 40(1991), 145-160.

[7] M. R. Tasković, Minimax theorems on posets with applications, Math. Japonica, 35(1990), 805-816.

[8] M. R. Tasković, General convex functions, Math. Japonica, 37(1992), 367-372.

[9] M. R. Tasković, Transversal fuzzy sets, (to appear).

[10] M. R. Tasković, On transversal points, Math. Japonica, 38(1993), 445-450.

[11] M. R. Tasković, Characterization of inductive posets with applications, Proc. Amer. Math. Soc., 104(1988), 650-659.

[12] M. R. Tasković, Theory of transversal point, spaces and forks, Monographs of a new theory, Beograd 2003, 1000 pages, to appear in Serbian.

[13] T. Terano, K. Asai and M. Sugeno, Fuzzy Systems Theory and its Applications, Academic Press, Inc., 1992.

[14] L. A. Zadeh, Fuzzy Sets, Information and Control, 8(1965), 338-353.

[15] Z. Wang and G. J. Klir, Fuzzy measure theory, New York and London, Plenum Press, 1992.

[16] H.-J. Zimmermann, Fuzzy set theory and its applications, Kluwer, Boston, 1991.

\author{
MATEMATIČKi FAKUltet \\ 11000 Beograd, P.O. Box 550 \\ Serbia \& Montenegro \\ RECEIVED JANUARY 20, 2002. \\ E-mail address: tassneza@eunet.yu
}

